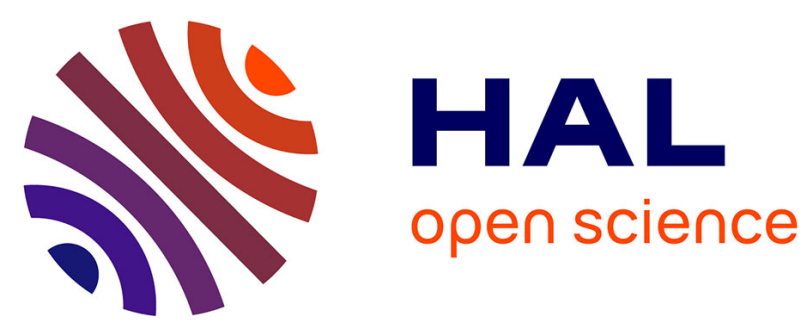

\title{
Modeling of cluster organization in metal-doped oxide glasses irradiated by a train of femtosecond laser pulses
}

Evgeniya Smetanina, Benoit Chimier, Yannick Petit, Nadezda Varkentina, Evelyne Fargin, Lionel Hirsch, Thierry Cardinal, Lionel Canioni, Guillaume Duchateau

\section{To cite this version:}

Evgeniya Smetanina, Benoit Chimier, Yannick Petit, Nadezda Varkentina, Evelyne Fargin, et al.. Modeling of cluster organization in metal-doped oxide glasses irradiated by a train of femtosecond laser pulses. Physical Review A : Atomic, molecular, and optical physics [1990-2015], 2016, 93 (1), pp.013846. 10.1103/PhysRevA.93.013846 . hal-01269838

\section{HAL Id: hal-01269838 \\ https://hal.science/hal-01269838}

Submitted on 14 Jan 2021

HAL is a multi-disciplinary open access archive for the deposit and dissemination of scientific research documents, whether they are published or not. The documents may come from teaching and research institutions in France or abroad, or from public or private research centers.
L'archive ouverte pluridisciplinaire HAL, est destinée au dépôt et à la diffusion de documents scientifiques de niveau recherche, publiés ou non, émanant des établissements d'enseignement et de recherche français ou étrangers, des laboratoires publics ou privés. 


\title{
Modeling of cluster organization in metal-doped oxide glasses irradiated by a train of femtosecond laser pulses
}

\author{
Evgeniya Smetanina, ${ }^{1}$ Benoit Chimier, ${ }^{1}$ Yannick Petit, ${ }^{1,2}$ Nadezda Varkentina, ${ }^{1}$ Evelyne Fargin, ${ }^{2}$ Lionel Hirsch, ${ }^{3}$ \\ Thierry Cardinal, ${ }^{2}$ Lionel Canioni, ${ }^{1}$ and Guillaume Duchateau ${ }^{1}$ \\ ${ }^{1}$ University of Bordeaux, CNRS, CEA, CELIA, UMR 5107, 351 Cours de la Liberation, 33405 Talence Cedex, France \\ ${ }^{2}$ University of Bordeaux, CNRS, ICMCB, UPR 9048, 87 avenue du Dr. A. Schweitzer, 33608 Pessac cedex, France \\ ${ }^{3}$ University of Bordeaux, CNRS, IMS, UMR 5218, 351 Cours de la Liberation, 33405 Talence Cedex, France
}

(Received 22 July 2015; revised manuscript received 14 December 2015; published 22 January 2016)

\begin{abstract}
The formation of silver cluster structures at submicrometer spatial scales under the irradiation by high-power femtosecond laser pulses with high repetition rate was observed in various glasses containing silver ions. In order to account for the formation of these structures in metal-doped glasses, we present a theoretical model for the organization of noble metallic clusters induced by a train of femtosecond laser pulses. The model includes photoionization and laser heating of the sample, diffusion, kinetic reactions, and dissociation of metallic species. This model was applied to reproduce the formation of cluster structures in silver-doped phosphate glass. The parameters of the silver structures were obtained numerically under various incident pulse intensities and number of pulses. Numerical modeling shows that the involved microscopic physical and chemical processes naturally lead to the emergence of a silver cluster organization, together with charge migration and subsequent trapping giving rise to a strong static electric field buried in the irradiated area as experimentally observed. Based on this modeling, a theoretical basis is provided for the design of new metallic cluster structures with nanoscale size.
\end{abstract}

DOI: 10.1103/PhysRevA.93.013846

\section{INTRODUCTION}

New optical functionalities created by direct laser writing (DLW) are among the impressive developments in modern optics. DLW based on high-repetition-rate femtosecond laser systems with high average powers is used to create alloptical switchers and other innovative photonics elements $[1,2]$. Femtosecond laser processing of glasses doped with photoactive agents (metallic ions) provides the possibility for controllable formation of color centers, metallic particles, and structures of submicron size in the bulk glass [3-11]. Fluorescence of metallic clusters [5], surface resonances of metallic nanoparticles [12-14], and effective second-order nonlinear response of glass assisted by metallic structures [6,7] are promising tools for high-resolution optical data storage technologies.

During DLW in photosensitive glasses doped by noble metal $(\mathrm{Cu}, \mathrm{Ag}, \mathrm{Au})$ laser pulse can induce the nucleation of the metallic ions and further cluster formation, which seems to be a universal clusterization process [9] under appropriate reduction-oxidation (redox) conditions. Indeed, the relation between the glass redox potential, the possible redox species of the considered metallic ions, the laser-induced ionization rate, and the strength of the thermal heating define the possibility of the metallic cluster formation in the bulk medium [15].

During the last years, a strong effort thus has been devoted to develop high-quality silver-containing optical glass materials, namely, based on a zinc phosphate glass matrix, as well as their laser structuring, which has led to the formation of highly fluorescent silver clusters and correlated nonlinear optical properties [3-6,9,16,17]. Figure 1 reports on a highly similar behavior in two commercial glassy matrices in which we had injected silver ions by thermal poling, namely, a microscope slide sodocalcique glass and a SIMAX borosilicate glass, respectively (Fig. 1). These breakthrough observations in adapted commercial glasses with broadband industrial applications suggest the universality of the laser-induced processes in systems containing noble metallic ions. Such a universal behavior strongly motivates the improvement of the understanding of the involved coupled phenomena, as those already studied in the silver-containing zinc phosphate glass. Indeed, this matrix has shown to have the appropriate properties for such DLW by the high-repetition-rate highpower near-IR femtosecond laser without additional annealing of the glass sample [6-8,17].

The phenomenology of this process is relatively complicated. The interplay between various microscopic physical and chemical mechanisms, associated to different time scales, gives rise to the emergence of a mesoscopic behavior taking place on submicrometer spatial scales, ultimately leading to a cluster organization in the glass. The resulting organized pattern is hereafter referred to as metallic nanostructures. The fastest process is ionization of glass during the femtosecond time scale of the laser pulse. The created free electrons diffuse, recombine with holes, and get trapped by the glassy network and metallic ions [15]. In the same time, electrons absorb the laser energy, which is transferred later into the lattice through collisions. If the characteristic time for thermal diffusion is longer than the interval between two successive pulses, the glass temperature increases during the pulse train, and the diffusion of metallic species can be thermally activated. At the same time, kinetic reactions produce metallic clusters, which may also be photodissociated in the region where the intensity of the next laser pulse is higher. Thus, cumulative creation of clusters takes place at the periphery of the beams, where the laser intensity and laser-induced temperature of the glass are low enough to prevent from dissociation, but still in the area of the beam where ionization and redox processes are launched. The laser-induced redistribution of metallic species provides a stabilized space charge separation inside the glass [8]. Local nonlinear properties are thus written in the glass, correspondingly to the electro-optical effect that arises 

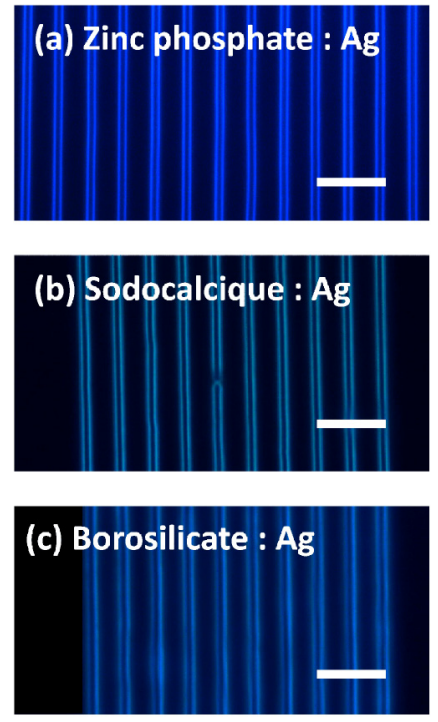

FIG. 1. Microscope fluorescence imaging (excitation at $375 \mathrm{~nm}$ ) of laser-induced silver clusters in three distinct glass matrices, highlighting the universal behavior of femtosecond direct laser writing in silver-containing glass matrices: (a) previously studied zinc phosphate glass [3-6,9,16,17]; report here of the similar formation of silver cluster structures (b) in a sodocalcite glass, and (c) in a borosilicate glass [18], both prepared by silver injection by thermal poling, similarly to what is described elsewhere [19]. Scale bar is $20 \mu \mathrm{m}$ in each case.

from the formation of a static electric field $E_{\mathrm{dc}}$ associated to the submicron nanoclusters formation. Such a static electric field creates locally an effective second-order susceptibility whereas it is zero before any irradiation of the glass (due to considerations on the lattice symmetry). Locally induced second harmonic generation is thus possible with various promising applications.

In silver-ion-doped glasses, silver species that appear after ionization process $\mathrm{Ag}^{0}, \mathrm{Ag}^{2+}$ together with silver ions $\mathrm{Ag}^{+}$ react to form the first stage of stable silver cluster $\mathrm{Ag}_{2}{ }^{+}$and further clusterization stages $\mathrm{Ag}_{m}{ }^{x+}, m>2$ [20,21]. In the case of an axially symmetric input laser beam and high enough input intensity, the clusterization process provides the formation of ring-shaped silver nanostructures. The associated space charge separation in silver nanostructures creates a frozen electric field $E_{\mathrm{dc}}$ with cylindrical symmetry. The value of the static electric field $E_{\mathrm{dc}}$ is very high and reaches up to $\sim 10^{9} \mathrm{~V} / \mathrm{m}$, almost corresponding to the electrical breakdown of the material, which allows the appearance of an effective second-order nonlinearity $\chi_{\text {eff }}^{(2)}=3 E_{\mathrm{dc}} \chi^{(3)}$ with values about $\sim 1 \mathrm{pmV}^{-1}[6,8,17]$. Such a second-order response forms a one- [8] or two-ring [6,17] shape, being coaxial with the fluorescent silver nanostructures [3-6].

To provide a clear understanding of the nanostructure formation and of the space charge separation process, there is a need to model the described-above laser-triggered kinetic reactions, diffusion, and dissociation of metallic species, together with the pulse-to-pulse laser heating of the sample. Such a theoretical development is addressed in this paper. We present the physical model and the associated numerical simulation of metallic nanostructure formation under a irradiation by train of femtosecond laser pulses. The heat diffusion equation with a laser-induced source term is coupled to the diffusion and kinetic equations of silver species through the thermal dependencies of the diffusion coefficients and kinetic rates according to the Arrhenius law. In turn, the resulting pulse-to-pulse electric field, calculated according to Gauss's law, plays a role as an electric field driving force in the diffusion of charged species, which is included in the derived diffusion equation. Thus, the complex physical and chemical effects are coupled in time and space.

Although the present model is developed for silver-doped phosphate glass, it can be generally applied to other photosensitive glasses. As it was demonstrated experimentally (Fig. 1) for silver-containing sodocalcique, borosilicate, and zinc phosphate glasses, the clusterization process of silver ions and further silver nanostructures formation exhibit a universal behavior for all considered glasses.

Simulations were performed for silver-doped phosphate glass. The model quantitatively reproduces the parameters of silver cluster radial distribution, that was observed experimentally in previous works [5]. The numerically obtained dependencies of the radius $R$ of ring nanostructure on the input laser pulse intensity and number of laser pulses in a train are in good agreement with the experimental observations. Based on a preliminary study, it is also able to describe the appearance of a frozen electric field that corresponds to the space redistribution of charged silver species which leads to the local breaking of the centrosymmetry, enabling direct laser poling of the irradiated glass, and thus the local creation of the second-order nonlinear optical response.

The paper is organized as follows: Section II presents in details the full model; Sec. III includes the used parameters to define the diffusion, kinetic, and dissociation processes. It also includes the main results obtained using the full model and a simplified form, that can be used for defined purposes under several assumptions, and the comparison of numerical results and experimentally observed data. Finally, the discussion and possible model predictions followed by the conclusion are given in Sec. IV.

\section{PHYSICAL MODEL}

\section{A. Phenomenological description of the silver cluster formation and general model}

Going from the general description of the laser-induced nanostructure formation in glass to the detailed description of the physical model we used, let us start with the schematically presented process of silver ring structure formation in glass (Fig. 2). First, free electrons are promoted during the laser pulse: yellow circle corresponds to the radial distribution of the free electrons in Fig. 2(a). In the area of maximal concentration of free electrons [green circle in Fig. 2(a)], the $\mathrm{Ag}^{0}$-formation kinetic reaction $\left(\mathrm{Ag}^{+}+e^{-} \rightarrow \mathrm{Ag}^{0}\right)$ begins according to the dependence of the kinetic reaction rate on the components' concentrations and the presence of electron diffusion. Note that there is a difference between a real metal atom $\mathrm{Ag}^{0}$ and a pair $\left(\mathrm{Ag}^{+}+e^{-}\right)$composed of an electron that is localized near a silver ion, without fully belonging to it, but being 


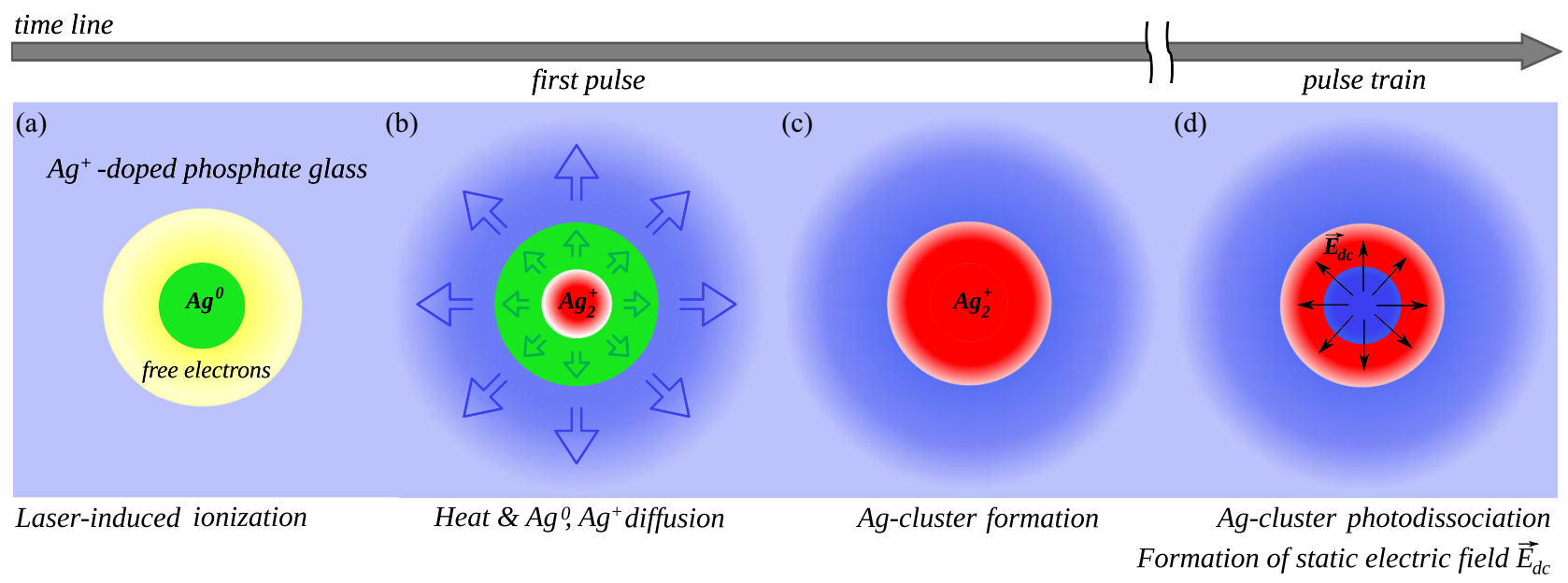

FIG. 2. Scheme of physical processes involving during the laser-induced nanostructure formation in silver-doped glass.

shared between surroundings. The second scenario is most probably realized under our considered conditions. Thus, in the modeling, $\mathrm{Ag}^{0}$ defines the pair of silver ion and trapped electron $\mathrm{Ag}^{+}+e^{-}$.

During the $\mathrm{Ag}^{0}$ formation, holes are trapped by silver ions $\mathrm{Ag}^{+}: h^{+}+\mathrm{Ag}^{+} \rightarrow \mathrm{Ag}^{2+}$ and the product of this trapping process is assumed not to be mobile. Indeed, $\mathrm{Ag}^{2+}$ should be considered rather as a hole trapped next to a silver ion $\mathrm{Ag}^{+}$ than a purely doubly ionized silver ion. Thus, the mobility of such a pair is negligible compared to the mobility of $\mathrm{Ag}^{+}$, due to its delocalization around the phosphate chains that form the glass network. These fast processes of charge trapping compete with different recombination processes $\left(e^{-}+h^{+}, h^{+}+\mathrm{Ag}^{0}\right.$, $\left.e^{-}+\mathrm{Ag}^{2+}\right)$. The concentration of $\mathrm{Ag}^{2+}$ is not presented in Fig. 2 to simplify the illustration.

The reaction $e^{-}+\mathrm{Ag}^{+} \rightarrow \mathrm{Ag}^{0}$ terminates when the concentration of electrons vanishes. At this moment, the distribution of $\mathrm{Ag}^{0}$ qualitatively corresponds to the slightly diffused distribution of previously existing free electrons [green circle, Fig. 2(b)]. The absorbed laser pulse energy induces an increase in the temperature in the on-axis region and is followed by heat diffusion [blue arrows, Fig. 2(b)]. The diffusion of the $\mathrm{Ag}^{0}$ [green arrows, Fig. 2(b)] is strongly activated by the temperature, which allows this species to meet the $\mathrm{Ag}^{+}$ ions and, at the same time to take part into the following kinetic reaction: $\mathrm{Ag}^{0}+\mathrm{Ag}^{+} \rightarrow \mathrm{Ag}_{2}{ }^{+}$. The small red circle [Fig. 2(b)] reproduces the beginning of this reaction in the area of maximal concentration of $\mathrm{Ag}^{0}$. The reaction of firststage silver cluster formation $\mathrm{Ag}^{0}+\mathrm{Ag}^{+} \rightarrow \mathrm{Ag}_{2}{ }^{+}$is finished when the concentration of silver atoms $\mathrm{Ag}^{0}$ vanishes. So, the distribution of $\mathrm{Ag}_{2}{ }^{+}$qualitatively corresponds to the slightly diffused distribution of previously existed $\mathrm{Ag}^{0}$ [red circle, Fig. 2(c)].

Because of fast trapping processes and associated kinetic reactions, the concentrations of free electrons, holes, and intermediate component $\mathrm{Ag}^{0}$ vanish before the next pulse. The next laser pulse destroys silver clusters through a photodissociation process in the on-axis region, where the laser pulse intensity is sufficiently high. Together with that, the laser-induced heating of the sample may prevent from the formation of clusters in the region where the temperature is high enough. In the same time, the next laser pulse creates the free electrons' population, that gives rise to kinetic reactions of cluster formation. The above-described cycle repeats from pulse to pulse, and the undissociated silver clusters are accumulated on the periphery of the beam forming the ring-shape structure [3-6] after the irradiation by a train of laser pulses [Fig. 2(d)]. Fast-diffusing free electrons destroy the initial spatial charge neutrality of the electronhole plasma in the laser focal spot and create an ambipolar electric field. Further trapping of electrons and holes provides the formation of frozen charge redistribution in space, and corresponding radial static electric field $\vec{E}_{\mathrm{dc}}$ [black arrows in Fig. 2(d)].

The present general model includes the equations for the evolution of temperature $T$, electrons concentration $n_{e}$, holes concentration $n_{h}$, and metallic species concentrations $n_{M}$ describing the clusterization process. For different glasses and doping metallic ions, only the kinetic equations should be changed according to the chemical processes involved in the metallic ion clusters formation. The model can be presented as a general form:

$$
\begin{gathered}
\frac{\partial T}{\partial t}=\Gamma_{D_{t h}}+S_{t h}, \\
\frac{\partial n_{e}}{\partial t}=\Gamma_{D}\left(T, n_{e}\right)+\Gamma_{M}\left(T, n_{e}, \vec{E}_{\mathrm{dc}}\right) \\
+\Gamma_{K}\left(n_{e}, n_{h}, n_{M}\right)+\Gamma_{I}+\Gamma_{D i s s}\left(n_{M}\right), \\
\frac{\partial n_{h}}{\partial t}=\Gamma_{K}\left(n_{h}, n_{e}, n_{M}\right)+\Gamma_{I}, \\
\frac{\partial n_{M}}{\partial t}=\Gamma_{D}\left(T, n_{M}\right)+\Gamma_{M}\left(T, n_{M}, \vec{E}_{\mathrm{dc}}\right) \\
+\Gamma_{K}\left(n_{M}, n_{e}, n_{h}\right) \pm \Gamma_{D i s s}\left(n_{M}\right), \\
\nabla \vec{E}_{\mathrm{dc}}=\frac{Q\left(n_{M}, n_{e}, n_{h}\right)}{\varepsilon_{0} \varepsilon_{\mathrm{dc}}}
\end{gathered}
$$

where $\varepsilon_{0}$ and $\varepsilon_{\mathrm{dc}}$ are vacuum and relative permittivities. $\Gamma_{D_{t h}}$ and $S_{t h}$ in Eq. (1) are the heat diffusion term and the laser-induced heat source term, respectively. In Eqs. (2) and (4), $\Gamma_{D}(T, n)$ and $\Gamma_{M}\left(T, n, \vec{E}_{\mathrm{dc}}\right)$ represent the diffusion 
and the mobility terms of involved components. Since the hole mobility is generally much lower than the electron mobility in dielectric media [22,23], it is assumed that the hole diffusion and mobility terms can be neglected in Eq. (3). $\Gamma_{K}$ includes the kinetic reactions of metallic species, and $\Gamma_{I}$ is the laser-induced electrons and holes source term in Eqs. (2) and (3). $\Gamma_{D}(T, n)$ and $\Gamma_{M}(T, n)$. The dissociation term $\Gamma_{\text {Diss }}\left(n_{M}\right)$ corresponds to the dissociation pathways of the involving metallic clusters. Its sign is negative if the metallic species is the dissociating cluster, and it is positive if the species is the product of the dissociation. In Eq. (5), $Q$ is the self-induced electric charge redistribution, and $\vec{E}_{\mathrm{dc}}$ describes the corresponding static electric field that builds up from pulse to pulse.

In the particular case of silver-doped glasses, the rate equations are presented for the silver ions $\mathrm{Ag}^{+}$, intermediate silver component $\mathrm{Ag}^{0}$, doubly ionized silver ions $\mathrm{Ag}^{2+}$, and silver clusters $\mathrm{Ag}_{2}{ }^{+}$concentrations. In this model, we do not consider further silver clusterization process and formation of larger silver clusters $\mathrm{Ag}_{m}{ }^{x+}, m>2$. This assumption is based on the fact that the $\mathrm{Ag}_{2}{ }^{+}$is a not mobile long-living cluster, that in general is the background for the further cluster growing. Thus, the distribution of the $\mathrm{Ag}_{2}{ }^{+}$already provides us relevant information about the area of larger silver cluster formation.

The mobility of zinc ions $\mathrm{Zn}^{2+}$ in phosphate glass (the conductivity of zinc-oxide containing phosphate glass is polaritonic [24]) is negligible in comparison to the mobility of silver ions $\mathrm{Ag}^{+}$under the temperature below the transition glass temperature $T_{g}$.

So, according to this general description, the metallic

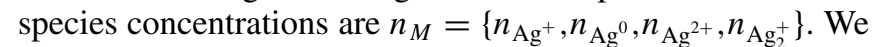
suppose that the $\mathrm{Ag}^{+}$and $\mathrm{Ag}^{0}$ are mobile, while the $\mathrm{Ag}^{2+}$ and $\mathrm{Ag}_{2}{ }^{+}$are not mobile. Equation (4) takes the following form:

$$
\begin{aligned}
& \frac{\partial n_{\mathrm{Ag}^{+}}}{\partial t}= \Gamma_{D}\left(T, n_{\mathrm{Ag}^{+}}\right)+\Gamma_{M}\left(T, n_{\mathrm{Ag}^{+}}\right) \\
&+\Gamma_{K}\left(n_{\mathrm{Ag}^{+}}, n_{e}, n_{h}, n_{\mathrm{Ag}^{0}}, n_{\mathrm{Ag}^{2+}}, n_{\mathrm{Ag}_{2}}\right) \\
&+\Gamma_{D i s s}\left(n_{\mathrm{Ag}_{2}}\right), \\
& \frac{\partial n_{\mathrm{Ag}^{0}}}{\partial t}=\Gamma_{D}\left(T, n_{\mathrm{Ag}^{0}}\right)+\Gamma_{K}\left(n_{\mathrm{Ag}^{0}}, n_{e}, n_{h}, n_{\mathrm{Ag}^{+}}, n_{\mathrm{Ag}^{2+}, n_{\mathrm{Ag}_{2}}}\right)
\end{aligned}
$$

$$
\begin{gathered}
\frac{\partial n_{\mathrm{Ag}^{2+}}}{\partial t}=\Gamma_{K}\left(n_{\mathrm{Ag}^{2+}}, n_{e}, n_{h}, n_{\mathrm{Ag}^{+}}, n_{\mathrm{Ag}^{0}}\right), \\
\frac{\partial n_{\mathrm{Ag}_{2}^{+}}}{\partial t}=\Gamma_{K}\left(n_{\mathrm{Ag}_{2}+}, n_{\mathrm{Ag}^{+}}, n_{\mathrm{Ag}^{0}}\right)-\Gamma_{\text {Diss }}\left(n_{\mathrm{Ag}_{2}+}\right) .
\end{gathered}
$$

During the evolution of involved species concentrations described by Eqs. (6)-(9), the total number and total charge of the components are conserved. The considered physical and chemical processes are detailed in the next subsections. Finally, they are all coupled and numerically integrated together according to the general description based on Eqs. (4) and (5).

\section{B. Absorption of the laser pulse energy}

Absorption of the laser pulse energy in general can be estimated using an approach that accounts for multiphoton ionization (MPI), impact ionization, and recombination
[25-30]. The laser-induced electron and hole source term for one pulse in Eqs. (1) and (2) is

$$
\Gamma_{I}=\sigma_{K}\left(\frac{I}{\hbar \omega}\right)^{K}\left(n_{a t}^{0}-n_{e}\right)+\sigma \frac{I}{U_{g}} n_{e}-\frac{n_{e}}{\tau_{r}},
$$

where $I(r, t, z)$ is the intensity distribution of the laser pulse. The first term corresponds to MPI process. The second term corresponds to the impact ionization process, where $\sigma$ is the inverse bremsstrahlung cross section, and the last term in Eq. (10) corresponds to the recombination process with the electron-hole recombination time $\tau_{r}$.

The intensity of the laser pulse decreases according to the equation

$$
\frac{\partial I}{\partial \zeta}=-\sigma_{K}\left(\frac{I}{\hbar \omega}\right)^{K}\left(n_{a t}^{0}-n_{e}\right) K \hbar \omega-\sigma I n_{e},
$$

where the input laser pulse intensity distribution $\left.I\right|_{\zeta=0}=$ $I_{0} \Phi(r) e^{-\tau^{2} / \tau_{0}^{2}}$ with $\tau_{0}=\tau_{p} / 2 \sqrt{\ln 2}, \tau_{p}$ is the laser pulse duration (FWHM), $\zeta \in\left[0, L_{\zeta}\right]$. The time in the frame moving with group velocity of the laser pulse $v_{g}$ is $\tau=v_{g} t-z$, and the position of the laser pulse in space, correspondingly to its central time slice $(\tau=0): \zeta=v_{g} t$.

Based on the absorption spectrum of the phosphate glass without silver ions [31,32], the band gap is typically estimated to be about $5 \mathrm{eV}$ [32]. For silver-doped phosphate glass, the band-gap value is estimated to be $4 \mathrm{eV}$ [33]. This decrease in the band gap is due to the appearance of states located in the band gap, induced by the presence of silver ions in the phosphate glass matrix. We thus consider the four-photon ionization process of Ag-doped phosphate glass with the cross section $\sigma_{4}=14.1 \times 10^{-118} \mathrm{~cm}^{8} \mathrm{~s}^{3}$, that was estimated based on the experimentally measured absorption [16]. The density of neutral atoms was taken as $n_{a t}^{0}=7.42 \times 10^{22} \mathrm{~cm}^{-3}$ [16]. We have checked that this value of the four-photon absorption cross section is comparable to predictions from the Keldysh's expression [25] in the considered range of intensities.

The experimentally measured absorption of such an ionized phosphate glass lies in a range from $0.04 \%$ to $0.5 \%$ of the incident laser pulse energy for pulse intensities from 2 to $8 \mathrm{TW} / \mathrm{cm}^{2}$. The corresponding estimation of the free-electron density is based on the absorption measurements and appears to be of the order of $10^{17} \mathrm{~cm}^{-3}$ [16]. The small values of the electron density and absorption indicate that impact ionization and subsequent electron avalanche are negligible. That is also in agreement with the absence of dielectric breakdown in the considered laser-matter interaction process.

Solving the system of equations (11) and (10) during the propagation of the strongly focused laser pulse in the glass along the Rayleigh length under the above-described conditions, without the avalanche term to account for experimental observations, the recombination time $\tau_{r}$ is found to be about 200 fs to account for both observed electron density and absorption.

To simplify the modeling, we set the electron and hole source term after each laser pulse to $\overline{n_{e}}(r)=n_{e}^{0} \Phi(r)^{K}$, where $n_{e}^{0}$ is a constant equal to $10^{17} \mathrm{~cm}^{-3}$ for all considered pulse intensities. Here, $\Phi(r)$ represents a normalized beam shape with radial symmetry, where $r$ is the radial coordinate and $K$ is the order of the multiphoton ionization process. Thus, for 
train of laser pulses, the electron and hole source term is

$$
\Gamma_{I}=\sum_{n=1}^{N_{p}} \overline{n_{e}}(r) \delta\left(t-n t_{L}\right),
$$

where $t_{L}=\frac{1}{v}, N_{p}$ is the number of laser pulses in a train, and $v$ is the laser repetition rate.

Finally, to estimate the absorption of the laser pulse intensity, we solve Eq. (11) with $n_{e}=\overline{n_{e}}(r)$ skipping the avalanche term.

The radial distribution of the absorbed energy density $S(r)$ is

$$
S(\vec{r})=\frac{1}{L_{\zeta}} \int_{-\infty}^{+\infty} I_{a b s}(r, \tau) d \tau,
$$

where $I_{a b s}(r, \tau)=\left.I(r, \tau)\right|_{\zeta=0}-\left.I(r, \tau)\right|_{\zeta=L_{\zeta}}$. The value of the absorbed energy $E_{a b s}$ is

$$
E_{a b s}=\int_{0}^{\infty} \int_{0}^{\infty} I_{a b s}(r, \tau) r d r d \tau .
$$

Note that we do not include the nonlinear effects as Kerr self-focusing and laser-induced plasma defocusing of laser pulse in the considering laser pulse propagation process because they do not play a key role: the strong focusing regime dominates self-focusing cubic nonlinearity and low plasma densities do not provide efficient defocusing of the pulse tail. Moreover, we assume that each laser pulse goes through the unmodified medium and thus the value of the absorbed energy for each laser pulse in the train remains the same. Such an assumption could be made because of relatively low changes of the refractive index (less than $10^{-3}$ in the present case [34]). Also, the possibility of the appearance of a new ionization pathways according to the creation of silver species $\left(\mathrm{Ag}^{0}, \mathrm{Ag}^{2+}, \mathrm{Ag}_{2}{ }^{+}\right)$is neglected because of the high-ionization potential: for $\mathrm{Ag}$ and $\mathrm{Ag}^{+}$it is 7.6 and $21.5 \mathrm{eV}$, respectively, and the ionization potential of silver clusters is of the same order.

An estimation of the absorbed energy and a comparison with the experimental values is provided in Sec. III B.

\section{Diffusion processes}

The temperature distribution in space and time is calculated according to

$$
\frac{\partial T}{\partial t}=D_{t h} \Delta T+\frac{1}{C_{p} \rho} \sum_{n=1}^{N_{p}} S(r) \delta\left(t-n t_{L}\right),
$$

where $S$ is the heat source term calculated according to Eq. (13), $C_{p}$ and $\rho$ are specific heat capacity and the density of the glass, respectively, $N_{p}$ is the number of laser pulses. The heat diffusion coefficient $D_{t h}$ in the glass is assumed to be constant because the temperature of the glass does not exceed the glass phase transition temperature and the glass remains in a solid state during the laser heating process. The first term on the right side accounts for the temperature diffusion $\Gamma_{D_{t h}}$ and the second term is the laser energy absorption term $S_{t h}$ in Eq. (1).

Diffusion $\Gamma_{D}$ and mobility $\Gamma_{M}$ terms of general model for silver ions $\mathrm{Ag}^{+}$and electrons $e^{-}$are coupled:

$$
\Gamma_{D}+\Gamma_{M}=\nabla(D \vec{\nabla} n)-\nabla\left(\mu \vec{E}_{\mathrm{dc}} n\right),
$$

where $D$ is the diffusion coefficient of the considered species, $n$ is its concentration, $\mu=\frac{q}{k T} D$ is the mobility in the electric field $\vec{E}_{\mathrm{dc}}$ defined according to the Einstein relation, and $q$ is the particle charge. For metallic species, the diffusion process depends on the lattice temperature $T$. To include thermally activated processes, the diffusion coefficient $D$ is expressed as

$$
D(T)=D\left(T_{0}\right) \exp \left(E_{a} / k T_{0}\right) \exp \left(-E_{a} / k T\right),
$$

where $k$ is the Boltzmann constant and $E_{a}=0.8 \mathrm{eV}$ is the activation energy [35].

Note that the Debye length for an electron density of $10^{17} \mathrm{~cm}^{-3}$ and a temperature of $0.1 \mathrm{eV}$ (estimated by a twotemperature model [22]) is of the order of $10 \mathrm{~nm}$. To calculate the static electric field due to the charge separation process, we account for all charged particle densities (participating in the considered process) with a spatial resolution of at least $0.5 \mathrm{~nm}$, thus significantly smaller than the Debye length. In addition, the electron diffusion length during the recombination time (roughly $200 \mathrm{fs}$ ) is of the order of $1 \mathrm{~nm}$, thus shorter than the Debye length. The local charge is thus conserved and the electrostatic properties of the material are supposed to be well described under this framework.

The diffusion processes of the charged particles (metallic ions, electrons $e^{-}$) induce an the electric field $\vec{E}_{\mathrm{dc}}$ inside the glass sample. The electric field $\vec{E}_{\mathrm{dc}}$ is calculated according to Gauss's law [Eq. (5)]. The charge redistribution $Q$ is the sum of all charged species concentrations taking into account the sign and density of the charges. So, the mobility term in diffusion equations of mobile charged particles accounts for the influence of the static electric field $\vec{E}_{\mathrm{dc}}$ which builds up during the diffusion process of the mobile particles. For neutral metallic species $M^{0}$, only the diffusion term $\Gamma_{D}$ is taken into account since the mobility in the electric field $\mu$ is zero.

The charge redistribution $Q$ for the particular case of silverdoped phosphate glass is calculated according to

$$
\frac{Q}{q_{0}}=n_{\mathrm{Ag}^{+}}+2 n_{\mathrm{Ag}^{2+}}+n_{\mathrm{Ag}_{2}+}+n_{h}-n_{e}-n_{L^{-}},
$$

where $q_{0}$ is the absolute value of the electron charge. This equation takes into account the concentrations of silver ions $\mathrm{Ag}^{+}$, doubly ionized silver ions $\mathrm{Ag}^{2+}$, silver clusters $\mathrm{Ag}_{2}{ }^{+}$, electrons and holes, and the input negative charge of the phosphate glass matrix that corresponds to the input silver ions distribution: $n_{L^{-}}=n_{\mathrm{Ag}^{+}}^{0}$.

\section{Kinetic reactions}

In this section, we present the kinetic terms $\Gamma_{K}$ of the silver clusterization process that is used for the application of the general model. For considered silver-doped phosphate glass, the kinetic terms $\Gamma_{K}$ describe the electrons $e^{-}$, holes $h^{+}$, silver ions $\mathrm{Ag}^{-}$, complexes of silver ions with electrons $\mathrm{Ag}^{0}$, doubly ionized silver ions $\mathrm{Ag}^{2+}$, silver clusters $\mathrm{Ag}_{2}{ }^{+}$. First of all, high intensity of femtosecond laser pulse provides the photoionization of the glass. Appearing in this process, photoelectrons and holes are trapped by silver ions $\mathrm{Ag}^{+}$. So, there are three fast competitive processes after laser irradiation: electron-hole recombination, $\mathrm{Ag}^{+}+e^{-} \rightarrow \mathrm{Ag}^{0}$ and $\mathrm{Ag}^{+}+h^{+} \rightarrow \mathrm{Ag}^{2+}[20,21,36]$. As soon as $\mathrm{Ag}^{0}$ and $\mathrm{Ag}^{2+}$ appear, the opposite reactions of recombination of these 
"frozen" charges with free electrons and holes begin: $\mathrm{Ag}^{0}+$ $h^{+} \rightarrow \mathrm{Ag}^{+}, \mathrm{Ag}^{2+}+e^{-} \rightarrow \mathrm{Ag}^{+}$. Further chemical reactions of silver species provide the formation of the first stage of stable silver clusters: $\mathrm{Ag}^{+}+\mathrm{Ag}^{0} \rightarrow \mathrm{Ag}_{2}{ }^{+}[20,21,36]$. At the same time, charge-recombination reaction is possible also: $\mathrm{Ag}^{2+}+\mathrm{Ag}^{0} \rightarrow 2 \mathrm{Ag}^{+}$. For electrons and holes, $\Gamma_{K}$ in Eqs. (2) and (3) is

$$
\Gamma_{K}\left(n_{e}, \ldots\right)=-K_{r} n_{e} n_{h}-K_{r} n_{\mathrm{Ag}^{2+}} n_{e}-K_{\mathrm{Ag}^{0}} n_{\mathrm{Ag}^{+}} n_{e}
$$

and

$$
\Gamma_{K}\left(n_{h}, \ldots\right)=-K_{r} n_{e} n_{h}-K_{r} n_{\mathrm{Ag}^{0}} n_{h}-K_{\mathrm{Ag}^{2+}} n_{\mathrm{Ag}} n_{h} .
$$

Here, the first terms in both equations (19) and (20) are the same: electron-hole recombination process characterized by the recombination constant $K_{r}$ estimated as $K_{r}=1 /\left(\tau_{r} n_{e}^{0}\right)$. The second terms are electron $\mathrm{Ag}^{2+}$ or hole $\mathrm{Ag}^{0}$ recombinations. The last terms are the electron or hole trapping by silver ions characterized by thermally activated kinetic constants $K_{\mathrm{Ag}^{0}}$ and $K_{\mathrm{Ag}^{2+}}$.

In Eq. (6) describing the time evolution of $n_{\mathrm{Ag}^{+}}$,

$$
\begin{aligned}
\Gamma_{K}\left(n_{\mathrm{Ag}^{+}}, \ldots\right)= & -K_{\mathrm{Ag}^{0}} n_{\mathrm{Ag}^{+}} n_{e}-K_{A^{2+}} n_{\mathrm{Ag}^{+}} n_{h} \\
& -K_{\mathrm{Ag}_{2}}+n_{\mathrm{Ag}^{+}} n_{\mathrm{Ag}^{0}}+K_{r} n_{\mathrm{Ag}^{0}} n_{h} \\
& +K_{r} n_{\mathrm{Ag}^{2+}} n_{e}+2 K_{\mathrm{Ag}^{+}} n_{\mathrm{Ag}^{2+}} n_{\mathrm{Ag}^{0}},
\end{aligned}
$$

where the first and the second terms are the free electron and hole trapping, the third term corresponds to the $\mathrm{Ag}_{2}{ }^{+}$ formation, the fourth and fifth terms describe the hole $\mathrm{Ag}^{0}$ and electron $\mathrm{Ag}^{2+}$ recombination processes.

Similarly, in Eqs. (7)-(9),

$$
\begin{gathered}
\Gamma_{K}\left(n_{\mathrm{Ag}^{0}}, \ldots\right)=K_{\mathrm{Ag}^{0}} n_{\mathrm{Ag}^{+}} n_{e}-K_{\mathrm{Ag}_{2}}+n_{\mathrm{Ag}^{+}} n_{\mathrm{Ag}^{0}} \\
-K_{r} n_{\mathrm{Ag}^{0}} n_{h}-K_{\mathrm{Ag}^{+}} n_{\mathrm{Ag}^{2+}} n_{\mathrm{Ag}^{0}}, \\
\Gamma_{K}\left(n_{\mathrm{Ag}^{2+}}, \ldots\right)=K_{\mathrm{Ag}^{2+} n_{\mathrm{Ag}^{+}} n_{h}-K_{r} n_{\mathrm{Ag}^{2+}} n_{e}} \\
-K_{\mathrm{Ag}^{+}} n_{\mathrm{Ag}^{2+}} n_{\mathrm{Ag}^{0}}, \\
\Gamma_{K}\left(n_{\mathrm{Ag}_{2}+}, \ldots\right)=K_{\mathrm{Ag}_{2}+} n_{\mathrm{Ag}^{+}} n_{\mathrm{Ag}^{0}} .
\end{gathered}
$$

The thermal dependence of the kinetic constants is evaluated according to the Arrhenius equation with preexponential factor given by the collision theory [37]

$$
K_{\mathrm{Ag}}(T)=K_{\mathrm{Ag}}\left(T_{0}\right) \sqrt{T / T_{0}} e^{E_{a} / k T_{0}} e^{-E_{a} / k T} .
$$

This dependence is taken into account for reactions of silver species formation. The $K_{\mathrm{Ag}}$ means here each of the kinetic constants: $K_{\mathrm{Ag}^{0}}, K_{\mathrm{Ag}^{2+}}, K_{\mathrm{Ag}_{2}+}, K_{\mathrm{Ag}^{+}}$. Note that the temperature growth of kinetic constants $K_{\mathrm{Ag}^{2+}}, K_{\mathrm{Ag}_{2}}{ }^{+}$provides a decrease in the influence of $\mathrm{Ag}^{0}$ and $\mathrm{Ag}^{+}$diffusion on the final distribution of the $\mathrm{Ag}_{2}{ }^{+}$since the chemical reactions forming not-mobile products $\left(\mathrm{Ag}_{2}{ }^{+}, \mathrm{Ag}^{2+}\right)$ are fast enough to limit the diffusion time of mobile reactants $\mathrm{Ag}^{0}$ and $\mathrm{Ag}^{+}$.

Finally, it is important to mention that we do not take into account the relaxation processes of silver components: $\mathrm{Ag}_{2}{ }^{+} \rightarrow 2 \mathrm{Ag}^{+}+e^{-}, \mathrm{Ag}^{2+} \rightarrow \mathrm{Ag}^{+}+h^{+}$. Such processes are supposed to be important when the number of laser pulses is large $N_{p} \geqslant 10^{4}$ because of the laser-induced increase of the glass temperature, and corresponding increase of the kinetic constants of these processes.

\section{E. Dissociation of silver clusters}

The description of the particular case of laser-induced silver clusters' dissociation process used for the application of the general model is given in this section. In general, the dissociation of metallic clusters by high-intensity laser pulse is a thermally assisted process that depends on the ratio between the photon energy and the dissociation energies of different dissociation pathways of the clusters.

Thus, in our modeling, the dissociation coefficient $K_{\text {diss }}$ is a phenomenological parameter that allows us to account for the evolution of the silver cluster ring structure. Both the high temperature of the lattice and the high laser pulse intensity could be responsible for the decrease in the number of silver clusters in the on-axis region. On one hand, at sufficiently high temperatures (about $200 \mathrm{~K}$ lower than $T_{g}$ ) the thermal dissolution of silver clusters occurs, that is described by the interplay between the redox potential of the glass composition, ionization of the glass, and glass temperature in [15]. On the other hand, the photodissociation of silver clusters occurs under the high-intensity laser pulse irradiation [3]. As presented in the Appendix, this process can be described in terms of multiphoton (two or three photons depending on the size of silver cluster) dissociation. The behavior of such a photodissociation function is similar to that what we use in our simulations, but the steepness of the theoretical one is smaller. It could be explained by the influence of the lattice temperature on the cluster dissociation process. Thus, the joint dynamics of these processes, thermal dissolution, and photodissociation of silver clusters provides the effective decrease in cluster concentration in the on-axis region. The laser irradiation or the thermal annealing (up to $T_{g}$ ) of the cluster-containing glass sample do not provide such effective cluster dissociation if they contribute separately $[7,38]$.

Following the model assumption, where only the smallest silver cluster $\mathrm{Ag}_{2}{ }^{+}$is considered, we account for the dissociation of $\mathrm{Ag}_{2}{ }^{+}$using the next phenomenological approach. The photoassisted and temperature assisted dissociation process of silver cluster $\mathrm{Ag}_{2}{ }^{+}$is assumed to go according to the reaction

$$
\mathrm{Ag}_{2}{ }^{+}+\hbar \omega \rightarrow \mathrm{Ag}^{+}+\mathrm{Ag}^{+}+e^{-},
$$

when another pathway consists of the reaction [39]

$$
\mathrm{Ag}_{2}^{+}+\hbar \omega \rightarrow \mathrm{Ag}^{+}+\mathrm{Ag}^{0} \text {. }
$$

Our choice of reaction (26) had been done because in our modeling the $\mathrm{Ag}^{0}$ represents a pair of a silver ion and a trapped electron that does not belong to the silver $\left(\mathrm{Ag}^{+}+e^{-}\right)$. The comparison between the two dissociation pathways [Eqs. (26) and (27)] will be discussed in the Appendix.

The dissociation term in Eqs. (3), (6), and (9) reads as

$$
\Gamma_{\text {Diss }}=\alpha_{\text {Diss }} \sum_{n=1}^{N_{p}} K_{\text {diss }} n_{\mathrm{Ag}_{2}+}+\delta\left(t-n t_{L}\right),
$$

where $\alpha_{\text {Diss }}$ corresponds to the mass and charge conservation according to the dissociation pathway used $\left(\alpha_{\text {Diss }}=-1\right.$ for $\mathrm{Ag}_{2}{ }^{+}, 2$ for $\mathrm{Ag}^{+}$, and 1 for $e^{-}$). 
Taking into account the fact that the intensity distribution of the laser pulse corresponds to the energy deposition in the glass and the temperature distribution, the behavior of the dissociation function $K_{\text {diss }}(r)$ is supposed to be a "steplike" function according to the multiphoton order of the photodissociation process. For sufficiently high laser pulse intensity, in the on-axis region of the Gaussian beam, the value of $K_{\text {diss }}(r)$ is close to unity, meaning that all silver clusters will be destroyed. On the periphery of the beam, the value of the dissociation function $K_{d i s s}(r)$ is close to zero, and most of the clusters remain undestroyed. Thus, $K_{\text {diss }}(r)$ is phenomenologically presented as a function of the absorbed laser pulse energy by the dependence on the source term $S$ in the heat diffusion equation (15):

$$
K_{\text {diss }}(r)=1-\exp \left[-S(r)^{N} / S_{0}^{N}\right] .
$$

The two free parameters $S_{0}$ and $N$ describe the position and the steepness of the dissociation threshold. The discussion on the possible way of theoretical description of dissociation function and the reasons to use in our simulations this phenomenological approach are presented in the Appendix.

\section{F. Simplified model}

We have developed a simplified model of silver cluster formation to decrease the numerical calculation time, and thus to be able to reproduce the silver clusters' distribution under up to $10^{8}$ laser pulses. The simplifications have been made as follows.

The laser-induced increase in glass temperature $T$ provides an exponential increase of the kinetic constants $K_{\mathrm{Ag}_{2}+}, K_{\mathrm{Ag}^{2+}}, K_{\mathrm{Ag}^{0}}$ according to the Arrhenius law [Eq. (25)]. That gives rise to a competition between fast processes involved in silver cluster formation, and various charge recombination processes. To simplify the general model previously presented, the ratio of silver cluster number to the number of electrons that appear after one laser pulse: $R_{n}=n_{\mathrm{Ag}_{2}+}(r=0) / n_{e}^{0}, R_{n} \leqslant 1$ is fixed. This assumption gives us the possibility to skip certain kinetic equations as follows: the equations for electrons and holes can be reduced and the value of silver clusters $\mathrm{Ag}_{2}{ }^{+}$(and doubly ionized silver ions for keeping the conservation of charge) that appear after each laser pulse becomes $R_{n} \overline{n_{e}}$.

The dissociation process of $\mathrm{Ag}_{2}{ }^{+}$is included as it was described previously. The charge recombination process $e^{-}+\mathrm{Ag}^{2+} \rightarrow \mathrm{Ag}^{+}$is supposed to be fast enough that all electrons $e^{-}$appeared after cluster dissociation finally react with doubly ionized silver ions $\mathrm{Ag}^{2+}$, resulting in the charge recombination. The equations for silver ion concentration $n_{\mathrm{Ag}^{+}}$and silver cluster concentration $n_{\mathrm{Ag}_{2}}+$ under considered simplifications then are

$$
\begin{gathered}
\frac{\partial n_{\mathrm{Ag}^{+}}}{\partial t}=-2 \sum_{n=1}^{N_{p}} R_{n} \overline{n_{e}} \delta\left(t-n t_{L}\right)+2 \sum_{n=1}^{N_{p}} K_{d i s s} n_{\mathrm{Ag}_{2}}+\delta\left(t-n t_{L}\right), \\
\frac{\partial n_{\mathrm{Ag}_{2}+}}{\partial t}=\sum R_{n} \overline{n_{e}} \delta\left(t-n t_{L}\right)-\sum_{n=1}^{N_{p}} K_{d i s s} n_{\mathrm{Ag}_{2}}+\delta\left(t-n t_{L}\right) .
\end{gathered}
$$

\begin{tabular}{|c|c|c|c|c|c|c|}
\hline & \multirow[b]{2}{*}{$D\left(T_{0}\right)\left(\mathrm{m}^{2} \mathrm{~s}^{-1}\right)$} & & \multirow[b]{2}{*}{$T_{0}(\mathrm{~K})$} & \multirow[b]{2}{*}{$E_{a}(\mathrm{eV})$} & & $L_{D}(\mu \mathrm{m})$ \\
\hline & & & & & & $300 \mathrm{~K} \quad 500 \mathrm{~K}$ \\
\hline Heat & $8 \times 10^{-7}$ & [4] & & & & 0.57 \\
\hline $\mathrm{Ag}^{+}$ & $5 \times 10^{-16}$ & [42] & 530 & 0.8 & [35] & "Negligible" \\
\hline $\mathrm{Ag}^{0}$ & $5 \times 10^{-11}$ & [3] & 350 & 0.8 & [35] & $5 \times 10^{-4} \quad 0.23$ \\
\hline$e^{-}$ & $3 \times 10^{-6}$ & [43] & & & & 1.1 \\
\hline
\end{tabular}

TABLE I. Diffusion coefficients.

Note that the equation for doubly ionized silver ions $n_{\mathrm{Ag}^{2+}}$ can be neglected since the distribution of $\mathrm{Ag}^{2+}$ corresponds to the distribution of silver clusters $\mathrm{Ag}_{2}{ }^{+}$according to the charge conservation.

\section{RESULTS}

The presented model in the previous section is now used to simulate the evolution of temperature and species concentration distributions in the focal area of a laser beam. In case of radial symmetry of the laser beam, the solution is defined in 1D+1 dimensions: radial coordinate $r$ and time $t$.

\section{A. Parameters}

The parameters of the femtosecond laser pulses and silver-doped phosphate glass used in our simulations are close to the parameters used in the experiments [5]. For all presented simulations, the wavelength of the laser pulse is $\lambda_{0}=$ $1030 \mathrm{~nm}$ and the repetition rate is $v=10 \mathrm{MHz}$. The laser beam is supposed to have a Gaussian profile $\Phi(r)=e^{-r^{2} / a_{0}^{2}}$, and input laser pulse intensity distribution is $I=I_{0} e^{-r^{2} / a_{0}^{2}} e^{-\tau^{2} / \tau_{0}^{2}}$, where $\tau_{0}=\tau_{p} / \sqrt{\ln 2}$. The duration of the laser pulse is taken equal to $\tau_{p}=470 \mathrm{fs}$ (FWHM), and the beam waist is $a_{0}=1.2 \mu \mathrm{m}$. The input energy of laser pulses is varied in the range $E=25-300 \mathrm{~nJ}$. The Rayleigh length $L_{z}$ is $10 \mu \mathrm{m}$. The number of laser pulses $N_{p}$ is varied from 10 to $10^{8}$.

A silver-doped phosphate glass $40 \mathrm{P}_{2} \mathrm{O}_{5}-4 \mathrm{Ag}_{2} \mathrm{O}$ $55 \mathrm{ZnO}-1 \mathrm{Ga}_{2} \mathrm{O}_{3}(\mathrm{~mol} \%)$ [3], is considered, with a uniform density of silver ions $n_{\mathrm{Ag}^{+}}^{0}=10^{21} \mathrm{~cm}^{-3}$. The density of the phosphate glass is $\rho=2.74 \mathrm{~g} \mathrm{~cm}^{-3}$, the specific-heat capacity is $C_{p}=0.78 \mathrm{~J} \mathrm{~g}^{-1} \mathrm{~K}^{-1}$. The dc-dielectric permittivity $\varepsilon_{\mathrm{dc}}$ is taken equal to $1[40,41]$.

The diffusion coefficients of silver species are presented in Table I. To estimate the influence of each diffusion process on the evolution of the whole system, we calculated the characteristic parameter: the diffusion length $L_{D}=2 \sqrt{D t}$, where $t=0.1 \mu \mathrm{s}$ is the delay between two consecutive laser pulses (10 $\mathrm{MHz}$ repetition rate). In case of temperaturedependent diffusion coefficients of silver species, the diffusion length is calculated for input glass temperature and for temperature reached during the laser-induced heating of the glass by a train of laser pulses to describe the dynamics of the temperature-activated diffusion processes.

The heat diffusion coefficient $D_{t h}$ is assumed to be constant because the glass temperature $T$ remains below the glass phase transition temperature $T_{g} \simeq 685 \mathrm{~K}[6-8,16,17]$.

Mobility parameters of electrons $n_{e}$ and silver ions $n_{\mathrm{Ag}^{+}}$are presented in Table II. The length $L_{m o b}=\mu E_{\mathrm{dc}} \tau$ characterizes 
TABLE II. Mobility coefficients.

\begin{tabular}{lcc}
\hline & $\mu\left(\mathrm{m}^{2} \mathrm{~s}^{-1} \mathrm{~V}^{-1}\right)$ & $L_{\text {mob }}(\mu \mathrm{m})$ \\
\hline $\mathrm{Ag}^{+}$ & $3 \times 10^{-20}$ & "Negligible" \\
$e^{-}$ & $3 \times 10^{-5}$ & 100 \\
\hline \hline
\end{tabular}

the mobility of charged particles in the electric field and is calculated for $\tau=0.1 \mu \mathrm{s}$ and the electric field $10^{9} \mathrm{~V} / \mathrm{m}$ corresponding to the experimentally observed value $[6-8,17]$. The characteristic parameters $L_{D}$ and $L_{m o b}$ will be discussed in the next subsection to analyze the interplay between thermal diffusion, species diffusion, and drift of charged mobile species in self-induced static electric field.

The kinetic constants for the reactions of silver species formation $K_{\mathrm{Ag}^{0}}, K_{\mathrm{Ag}^{2+}}, K_{\mathrm{Ag}_{2}}+$ are presented in Table III, the recombination constant is defined as $K_{r}=1 /\left(\tau_{r} n_{e}^{0}\right)$, and $\tau_{r}$ is of the order of $200 \mathrm{fs}[20,21,36]$. For the slow recombination reaction $\mathrm{Ag}^{2+}+\mathrm{Ag}^{0} \rightarrow 2 \mathrm{Ag}^{+}$, the kinetic constant is supposed to be of the order $K_{\mathrm{Ag}^{+}}\left(T_{0}\right)=10^{-10} \mathrm{~cm}^{3} \mathrm{~s}^{-1}$, with $T_{0}=300 \mathrm{~K}$.

The characteristic time $\tau_{\mathrm{Ag}}$ of silver species $\mathrm{Ag}^{0}, \mathrm{Ag}^{2+}$, $\mathrm{Ag}_{2}+$ formation could be estimated as $\tau_{\mathrm{Ag}} \approx 1 /\left(K_{\mathrm{Ag}} n_{\mathrm{Ag}^{+}}^{0}\right)$, where $K_{\mathrm{Ag}}$ means here each of the kinetic constants for silver species formation that were mentioned above. The first column shows the product of the reaction, the second column presents the kinetic constant $K_{\mathrm{Ag}}$ for temperature $T_{0}=300 \mathrm{~K}$, the third and fourth columns contain the values of corresponding characteristic times $\tau_{\mathrm{Ag}}$ at temperatures $T=300$ and $400 \mathrm{~K}$, that will be discussed in the next subsection.

\section{B. General consideration}

Here, we provide a general consideration on the processes involved in the nanostructures formation. Let us analyze the evolution of the whole system described by the present model based on the characteristic parameters such as the value of absorbed energy of the laser pulse and the temperature increase during the pulse train, the diffusion lengths of the heat and diffusing species, the mobility lengths of charged diffusing species in the electric field, and the characteristic times of kinetic reactions.

To define the laser-induced source term in the thermal diffusion equation [Eq. (1)], the value of the laser-induced electron density $n_{e}^{0}$ is taken equal to $10^{17} \mathrm{~cm}^{-3}$ in Eq. (12), that is close to the experimentally measured value [16]. For considered input laser pulse intensities $I_{0}$ from 1 to $10 \mathrm{TW} / \mathrm{cm}^{2}$, that corresponds to pulse energies $E_{0}$ from 25 to $240 \mathrm{~nJ}$, the absorbed part of the input pulse energy

TABLE III. Kinetic constants.

\begin{tabular}{lcccc}
\hline \hline & & \multicolumn{2}{c}{$\tau_{\mathrm{Ag}}(\mathrm{ps})$} \\
\cline { 3 - 5 } & $K_{\mathrm{Ag}}\left(\mathrm{cm}^{3} \mathrm{~s}^{-1}\right)$ & & $300 \mathrm{~K}$ & $400 \mathrm{~K}$ \\
\hline $\mathrm{Ag}^{0}$ & $8 \times 10^{-11}$ & {$[20]$} & 12.5 & 0.005 \\
$\mathrm{Ag}^{2+}$ & $1 \times 10^{-10}$ & {$[21]$} & 10 & 0.004 \\
$\mathrm{Ag}_{2}{ }^{+}$ & $3.33 \times 10^{-12}$ & {$[36]$} & 300 & 0.12 \\
\hline \hline
\end{tabular}

is about $1 \%: E_{a b s} / E_{0} \in[0.04,0.8] \%$, which is close to the experimentally observed values of absorbed energy $[3,16]$. Thus, the absorbed energy is less than $1 \%$ of the input laser pulse energy. That provides a sufficiently strong glass heating process to activate species kinetics and diffusion, but, however, leads to a temperature of the glass under the phase transition temperature, i.e., no liquid phase may appear.

The evolution of radial distribution of temperature $T(r, t)$ is presented in Fig. 3 for input laser energy $E_{0}=150 \mathrm{~nJ}$ during 100 pulses, that corresponds to $10 \mu \mathrm{s}$ with the considered laser parameters. the initial condition for the thermal diffusion (15) is

$$
T(r, t=0)=T_{0}
$$

and boundary conditions are

$$
\left.\frac{\partial T(r, t)}{\partial r}\right|_{r=0}=0,\left.\quad T(r, t)\right|_{r=\infty}=T_{0}, \text { and } T_{0}=300 \mathrm{~K} .
$$

The high-temperature vertical line sequence in Fig. 3 illustrates the increase of the temperature according to the laser pulse sequence. Between two pulses, the temperature decreases due to the heat diffusion process. Because of the high-repetition rate and high-peak intensity of the laser pulses, heat diffusion on the periphery of the beam becomes smooth in time with no visible vertical lines, showing that on the periphery the heat source term could be considered as the continuous heat source. Note that the temperature remains below the glass transition temperature $T_{g} \simeq 685 \mathrm{~K} \mathrm{[16]}$.

In case of phosphate glass doped by silver ions, the initial conditions used to solve the diffusion equation (16) for all diffusing particles are zero except initially presented in the glass silver ions:

$$
\begin{aligned}
n_{e^{-}, \mathrm{Ag}^{0}}(r, t=0) & =0 \text { and } \\
n_{\mathrm{Ag}^{+}}(r, t=0) & =n_{\mathrm{Ag}^{+}}^{0},
\end{aligned}
$$

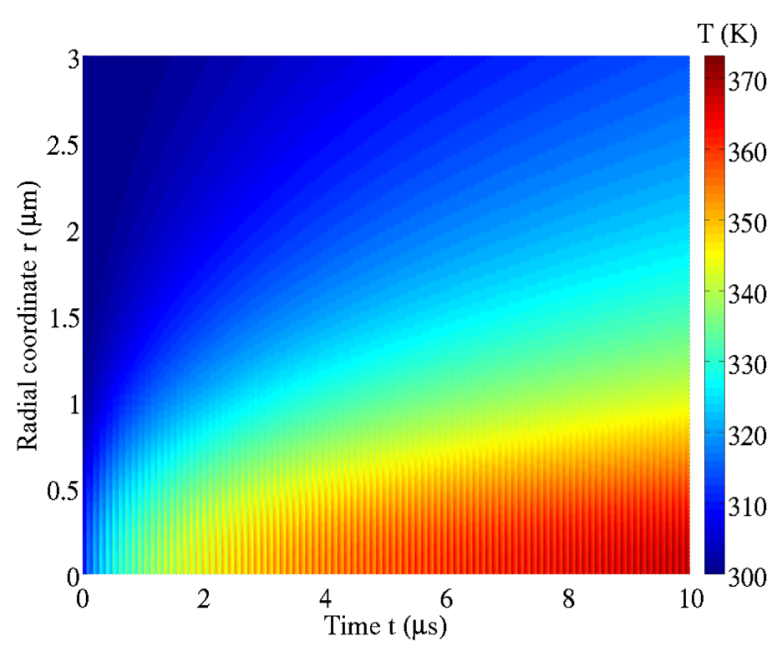

FIG. 3. Time evolution of radial distribution of the glass temperature $T(r, t)$ during laser heating process: $E_{0}=150 \mathrm{~nJ}, v=$ $10 \mathrm{MHz}, \tau_{p}=470 \mathrm{fs}, a_{0}=1.2 \mu \mathrm{m}, L_{z}=10 \mu \mathrm{m}$, and full time $10 \mu$ s corresponds to the 100 laser pulses. 
and boundary conditions are

$$
\begin{aligned}
&\left.\frac{\partial n_{e^{-}, \mathrm{Ag}^{+}, \mathrm{Ag}^{0}}(r, t)}{\partial r}\right|_{r=0}=0, \\
&\left.n_{\mathrm{Ag}^{+}}\right|_{r=\infty}=n_{\mathrm{Ag}^{+}}^{0},\left.\quad n_{e^{-}, \mathrm{Ag}^{0}}\right|_{r=\infty}=0 .
\end{aligned}
$$

The heat diffusion length $L_{h}=0.57 \mu \mathrm{m}$ is several orders larger than the diffusion lengths of silver species $L_{\mathrm{Ag}^{0}}, L_{\mathrm{Ag}^{+}}$ at the input temperature $T=300 \mathrm{~K}$ (Table I). According to the Arrhenius law, already at the temperature $T=500 \mathrm{~K}$ (that could be reached after $N_{p} \gtrsim 10^{4}$ ) $\mathrm{Ag}_{0}$ diffusion length increases up to few tenths of micron, and reaches enough large value to influence on the fast process of silver cluster formation (Table I).

The mobility of the charged diffusing particle is connected with the diffusion coefficient according to the Einstein relation. Mobility coefficient of electrons is taken equal to $\mu_{e}=3 \times 10^{-5} \mathrm{~m}^{2} \mathrm{~s}^{-1} \mathrm{~V}^{-1}[23,44]$. The estimation of the temperature of laser-induced electrons $T_{e}$ has been performed by the calculation of the part of incident laser pulse energy absorbed in self-induced laser plasma $[22,44,45]$. The value of the electron temperature $T_{e}$ reaches about $0.1 \mathrm{eV}$ under considered conditions. Taking into account this estimation of the electron temperature $T_{e}$, the diffusion coefficient of electrons is $D_{e}=3 \times 10^{-6} \mathrm{~m}^{2} \mathrm{~s}^{-1}$. In case of dielectric media, the laser-induced holes are supposed to have a negligible diffusion and mobility coefficients in comparison with the electrons [23]. At the same time, the mobility of silver ions also is much less than the mobility of electrons (Table II). The reason to take the silver ions mobility into account is that the electrons (and holes) exist only several hundred femtoseconds after the laser pulse and finally they recombine with each other or are trapped, while silver ions participate in the diffusion and drift processes during all time of the laser-matter interaction and their concentration is several orders larger than the electron and hole concentrations. The influence of the static electric field on the species redistribution is represented by the value of the mobility length $L_{m o b}$. Taking the electric field equal to $E_{\mathrm{dc}}=10^{9} \mathrm{~V} / \mathrm{m}$, that is close to the maximal observed experimental values [6-8,17], the mobility length of the electrons reaches such a large value $L_{m o b_{e}}=100 \mu \mathrm{m}$ taking a time scale of the order of the delay between two consecutive pulses. In presence of very high static electric field it was assumed that the electron drift velocity saturates similarly to the charge carriers drift velocity in semiconductors [46]. The value of the electric field that corresponds to the electron drift velocity saturation regime is equal to $E_{\mathrm{dc}}=10^{5} \mathrm{~V} / \mathrm{m}$ in our simulations. Thus, taking into account the saturation effect of the electrons drift in the strong electric field $E_{\mathrm{dc}}$, the mobility length of electrons $L_{m o b_{e}}$ does not exceed $0.01 \mu \mathrm{m}$.

Because of the laser-induced increase in the temperature $T$ with the corresponding increase of the kinetic constants $K_{\mathrm{Ag}}$, the characteristic time $\tau_{\mathrm{Ag}}$ of silver species $\mathrm{Ag}^{0}, \mathrm{Ag}^{2+}$, $\mathrm{Ag}_{2}+$ formation decreases during the laser-matter interaction process. At temperature $T=300 \mathrm{~K}$, the characteristic times $\tau_{\mathrm{Ag}}$ of the kinetic reactions are in the picosecond range, when at the temperature $T=400 \mathrm{~K}$, the characteristic times reach the femtosecond range. In that case, a strong competition between kinetic reactions of silver cluster formation and diffusion processes takes place.
During the first stage of the clusterization process, the two fastest processes compete: electron-hole recombination and electron trapping by silver ions. To describe this temperaturedependent competition, we can use the ratio between the reactions rates: $K_{r} n_{e} n_{h}$ and $K_{\mathrm{Ag}^{0}} n_{e} n_{\mathrm{Ag}^{+}}$. The cluster formation process is initiated by the formation of $\mathrm{Ag}^{0}$, which results from the electron trapping by silver ions. However, at room temperature, the electron-hole recombination process is dominant. As the kinetic constant $K_{\mathrm{Ag}^{0}}$ increases with the temperature [Eq. (25)], both processes begin to be comparable when the temperature is about $400 \mathrm{~K}$, which can be defined as the temperature threshold for cluster initiation. Note that this temperature is obtained after the first hundreds of pulses within our conditions, which is in a good agreement with experimental data showing that no significant fluorescent signal from clusters is observed for a number of pulses smaller than roughly 100 [5].

The second stage of the clusterization process is the formation of $\mathrm{Ag}_{2}{ }^{+}$. The kinetic rate of this process $K_{\mathrm{Ag}_{2}}+n_{\mathrm{Ag}^{0}} n_{\mathrm{Ag}^{+}}$ is higher than the rates of trapped charges recombination processes $K_{\mathrm{Ag}^{+}} n_{\mathrm{Ag}^{0}} n_{\mathrm{Ag}^{2+}}$ and $K_{r} n_{\mathrm{Ag}^{0}} n_{h}$. Here, the cumulative effect of the dissociation function $K_{\text {diss }}$, that will be described in the next section, limits the growth of clusters in the on-axis region after 100-1000 laser pulses depending on the energy of the laser pulse.

\section{Validation of the simplified model}

The reliability of the simplified model was confirmed by the comparison of the results obtained using full model and simplified model presented in Sec. II F. Figure 4 (top) shows the temperature distribution $T(r)$ calculated for a train of $10^{3}$ laser pulses with energy per pulse $200 \mathrm{~nJ}$. The maximal temperature reaches about $570 \mathrm{~K}$, that is lower than the phase transition temperature of the glass $T_{g}$. Figure 4 (bottom) depicts the $\mathrm{Ag}_{2}{ }^{+}$distribution as predicted by both full (solid
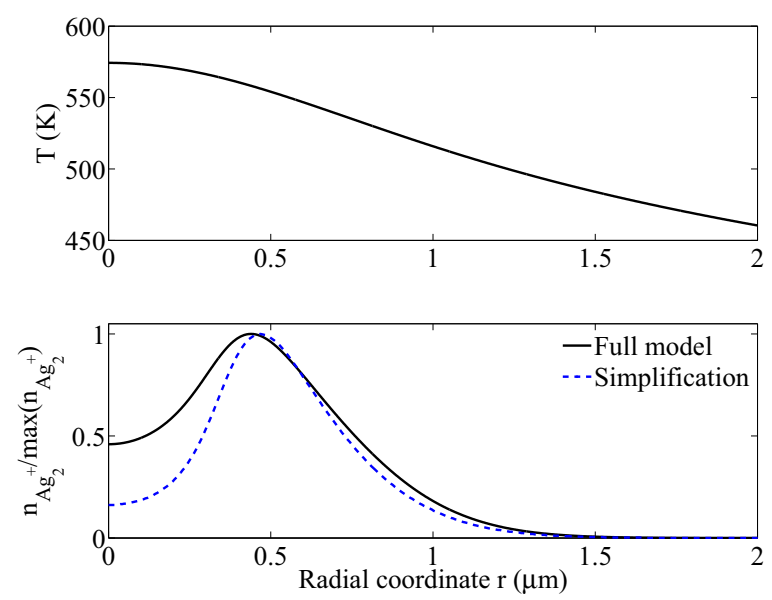

FIG. 4. (Top) Radial distribution of lattice temperature. (Bottom) Radial distribution of the silver cluster concentration $n_{\mathrm{Ag}_{2}+}$. Full model (black solid line) and simplified model (blue dashed line) predictions are provided. The input laser pulse energy is $E_{0}=200 \mathrm{~nJ}$, number of pulses is $N p=1000$. Dissociation function parameters are: $S_{0}=80, N=6$. 
line) and simplified (dashed line) models. For the latter, $R_{n}$ is set to 0.1 [see Eqs. (30) and (31)].

The simplified model reproduces the spatial distribution of silver cluster concentration. In the on-axis area, the amount of silver clusters is nonzero. According to the full model, it occurs because after the dissociation process in the on-axis region induced by the last laser pulse, the clusters still are allowed to grow and finally their concentration reaches a nonzero value. In case of simplified model, the recombination process of dissociated components with trapped holes is supposed to be so fast that no clusters appear because of reclusterization of dissociated components during time between pulses. Thus, the on-axis value of cluster concentration predicted by the simplified model is smaller than the value predicted by the full model and the slight shift between the maxima of the cluster distributions exists. The edges of the ring structure are smoother in case of full model prediction because of the temperature-activated diffusion process of silver species. The simplification of the model is focused mostly on the reproduction of the ring structure form, and does not imply a complete coincidence of the total number of clusters in the ring structure with the data obtained using the full model. The previous comparison leads us to conclude that the simplified model can be used for reconstruction of the silver nanostructures radius depending on the input pulse intensity and number of laser pulses in the train.

\section{Nanostructure formation}

The simplified model presented above is used to simulate the evolution of the radial distribution of nanostructures. To be able to reproduce the silver ring structure formation under up to $10^{8}$ laser pulses, we suppose that $R_{n}=10^{-4}$, where $R_{n} \overline{n_{e}}(r)$ is the number of silver atoms that appears after each laser pulse. Such an effective decrease of parameter $R_{n}$ describes the influence of the relaxation processes of $\mathrm{Ag}_{2}{ }^{+} \rightarrow 2 \mathrm{Ag}^{+}+e^{-}, \mathrm{Ag}^{2+} \rightarrow \mathrm{Ag}^{+}+h^{+}$, and compensates the possible underestimation of recombination processes in the full model. That is significant for a large number of pulses $\left(N_{p}>10^{4}\right)$. This $R_{n}$ value allows us to prevent from the saturation of silver components concentrations $n_{\mathrm{Ag}_{2}+}, n_{\mathrm{Ag}^{2+}}$ under a large number of laser pulses. That affords to reconstruct the dependencies of the radius of the silver ring structure $R$ on the input laser pulse intensity $I_{0}$ and number of laser pulses in the train $N_{p}$.

The free parameters of dissociation function were taken as $S_{0}=80, N=6$ [Eq. (29)] to obtain a good agreement with the experimental data as shown hereafter. Note that this choice does not depend on the laser intensity, neither the number of pulses. Furthermore, slight deviations from these parameters always provide a correct agreement. That confirms the reliability of the proposed modeling.

The heat source term $S(r)$ and dissociation function $K_{\text {diss }}(r)$ are presented in Fig. 5 for various input laser pulse energies. Increasing the input laser pulse energy, while the amount of absorbed energy increases, that provides higher values of the heat source term in Eq. (1) calculated according to Eq. (13) [Fig. 5 (top)]. For lower energies, the maximal value of the dissociation function $K_{d i s s}$ is less than 1, thus a number of pulses are needed to destroy most part of the
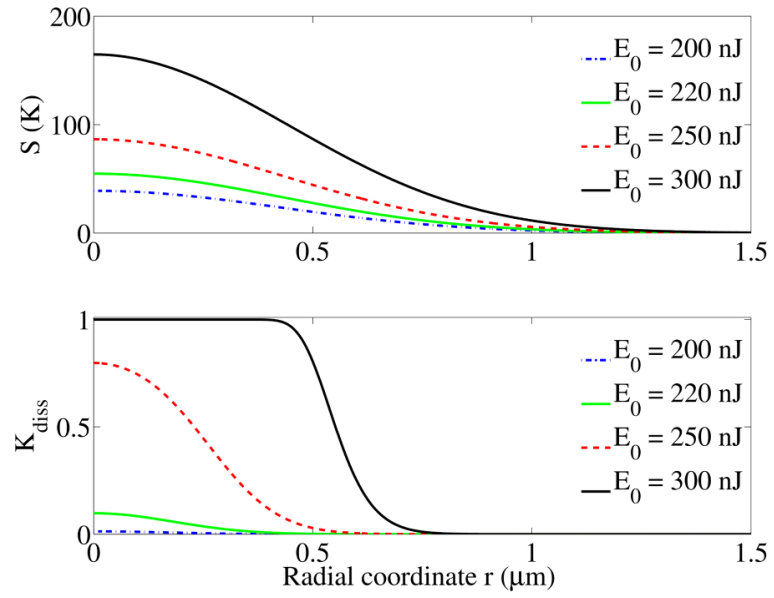

FIG. 5. (Top) Source terms in the heat diffusion equation $S(r)$ for input laser pulse energies $E_{0}=200,220,250$, and $300 \mathrm{~nJ}$, calculated according to Eq. (13). (Bottom) Corresponding dissociation function $K_{\text {diss }}(r)$ calculated according to Eq. (29). Dissociation function parameters are $S_{0}=80, N=6$.

clusters in the on-axis region. But, for sufficiently high energy, the values of $K_{\text {diss }}$ reach 1 in the on-axis region since all silver clusters are destroyed already after one pulse in this area. Such a shape of dissociation function $K_{\text {diss }}$ is in agreement with general description of probability of multiphoton dissociation processes. The discussion on possible theoretical approach to describe the dissociation process of silver clusters under considered conditions is presented in the Appendix.

Figure 6 presents the experimentally [5] (left column) and the numerically predicted (right column) silver cluster distribution for input laser pulse intensities $I_{0}=4.8,6,7.4$, $12 \mathrm{TW} / \mathrm{cm}^{2}$, and a number of pulses $N_{p}=10^{7}$. The distributions of numerically obtained silver clusters and experimentally observed (through fluorescence) are in good agreement. When the intensity is low $I_{0}=4.8 \mathrm{TW} / \mathrm{cm}^{2}$, the dissociation process of silver clusters in the on-axis region is weak and no good resolved ring structure can be found even after $10^{7}$ laser pulses [Figs. 6(d) and 6(h)]. With the increase of the input pulse intensity up to $I_{0}=6 \mathrm{TW} / \mathrm{cm}^{2}$, the dip in the silver cluster concentration is formed in the on-axis region because of the increase in the efficiency of the dissociation process [Figs. 6 (c) and $6(\mathrm{~g})]$. Further increasing of the incident laser intensity $I_{0}$ leads to the increase in the radius $R$ of the silver clusters' ring structure [Figs. 6(a), 6(b), 6(e), and 6(f)]. Note that the limited spatial resolution of the objective used for the recording of the silver clusters fluorescence blurs the real spatial distribution of the fluorescent signal that should be taken into account for the comparison of numerical obtained distributions of silver clusters, and its fluorescent signals detected experimentally.

The width of the numerically simulated $\mathrm{Ag}_{2}{ }^{+}$ring structure is about 200-300 nm, which is similar to what is observed experimentally $[3,5,9]$. The fluorescent signal detected in the experiments mostly corresponds to the large silver clusters. Thus, the difference in the experimentally and numerically estimated width of the ring structures can be explained by the fact that distribution of silver clusters with higher number of atoms should form thinner structure on the background distribution of centers of clusterization $\mathrm{Ag}_{2}{ }^{+}$. 


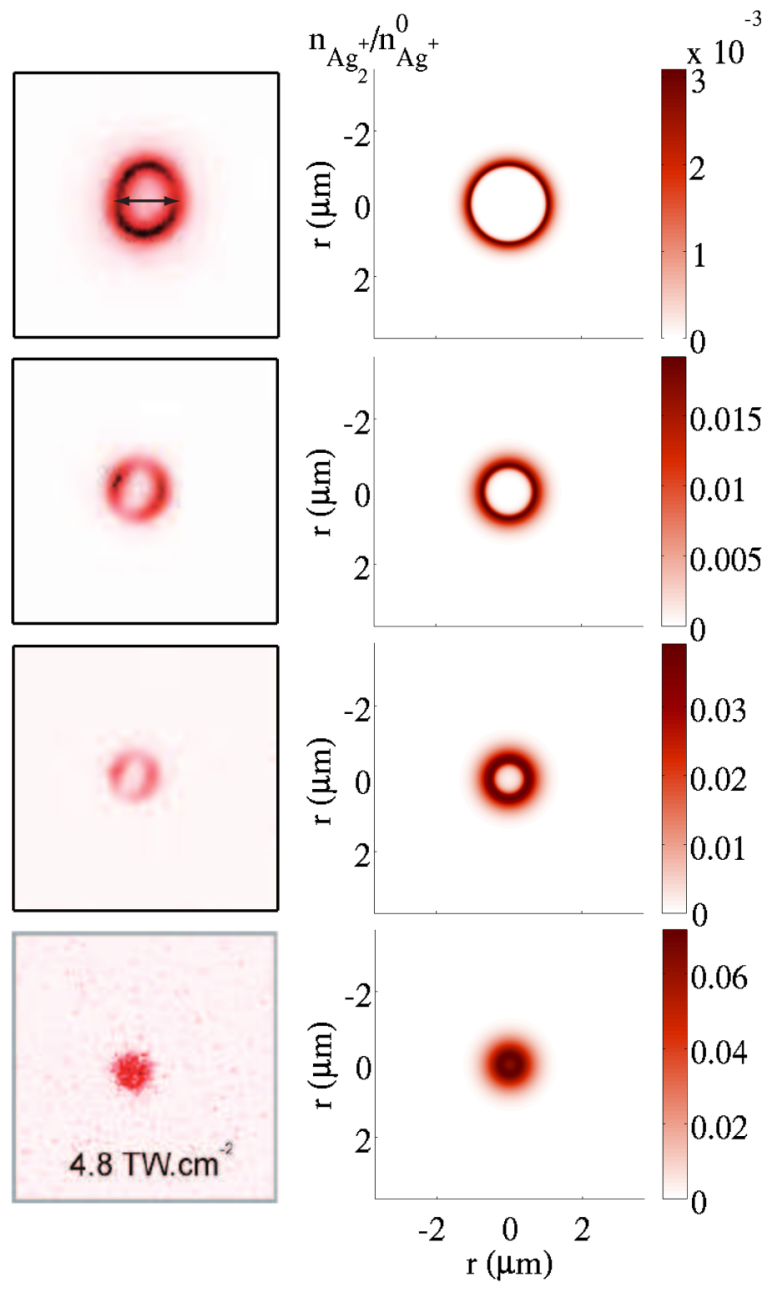

FIG. 6. Ring nanostructures of silver clusters for various input laser intensities $I_{0}=4.8,6,7.4,12 \mathrm{TW} / \mathrm{cm}^{2}$. First column (a), (b), (c), (d): experimentally observed fluorescent signal of silver clusters in phosphate glass [5]. The experimentally obtained value of the ring diameter is $\Delta x=1.96 \pm 0.3 \mu \mathrm{m}$ for $12 \mathrm{TW} / \mathrm{cm}^{2}$ (a). Second column (e), (f), (g), (h): numerically simulated radial distribution of the silver cluster concentration $n_{\mathrm{Ag}_{2}+}(r) / n_{\mathrm{Ag}^{+}}^{0}$. Number of pulses is $N_{p}=10^{7}$. Dissociation function parameters are $S_{0}=80, N=6$.

The dependence of silver structure ring radius $R$ on the input laser pulse intensity $I_{0}$ is presented for a given number of pulses $N_{p}$ from $10^{3}$ up to $10^{7}$ in Fig. 7. The experimental measurements of the radius values [5] are also presented. For a number of laser pulses, the calculated radius increases with the laser intensity. An intensity threshold of ring structure formation can be defined for a fixed number of pulses as the maximal laser intensity, for which it is no ring nanostructure yet. This intensity threshold is well reconstructed numerically. For $10^{3}$ laser pulses, the intensity threshold is $I_{0}=7.4 \mathrm{TW} / \mathrm{cm}^{2}$. There is no ring structure if the intensity is under the threshold. For lower input intensities $I_{0}$, the number of pulses $N_{p}$ influences more strongly on the ring structure radius $R$. For relatively high input intensities $I_{0}$, the radius of the ring structure $R$ does no longer strongly depend on the laser pulse number. Such a behavior of the ring structure radius depending on the input laser pulse intensity

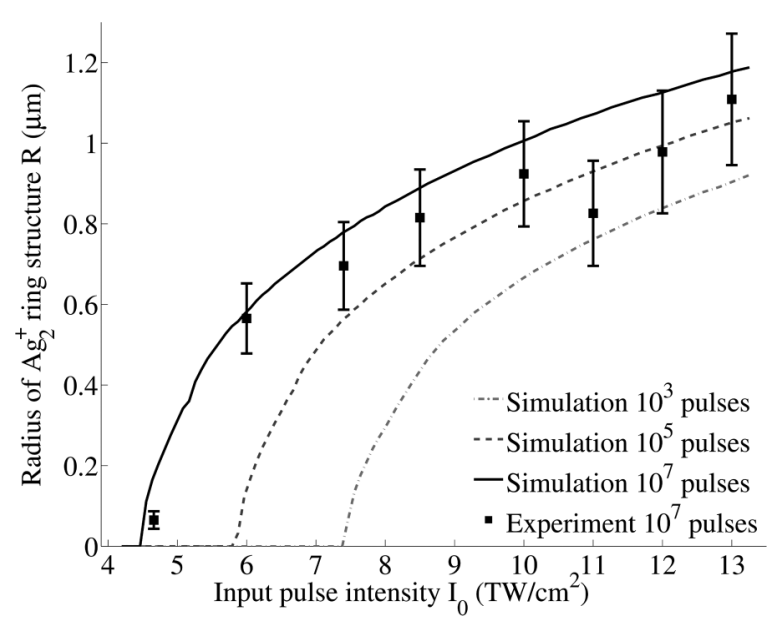

FIG. 7. Radius of ring nanostructure $R$ as a function of the input pulse intensity $I_{0}$ from experiments [5] and numerically predicted. Laser pulse parameters: input laser pulse intensity is varied in the range $I_{0}=4-13 \mathrm{TW} / \mathrm{cm}^{2}$, and corresponding energies are $E_{0}=$ 90-300 nJ. Numerical dependencies $R\left(I_{0}\right)$ are presented for various number of pulses $N_{p}=10^{3}, 10^{5}, 10^{7}$. Beam waist is $1.2 \mu \mathrm{m}$, pulse duration is $470 \mathrm{fs}$, and the repetition rate is $10 \mathrm{MHz}$. Dissociation function parameters are $S_{0}=80, N=6$.

and the number of laser pulses in a train is determined by the multiphoton dissociation process of silver clusters.

The theoretically obtained dependence of the ring structure radius on the number of pulses $N_{p}$ for input laser intensity $I_{0}=7.4 \mathrm{TW} / \mathrm{cm}^{2}$ is presented in Fig. 8 together with the experimental data [5]. By increasing the laser pulse's number, we can decrease the intensity threshold value needed to produce the ring structure. If the number of pulses is under $10^{3}$, and cumulative dissociation effect is weak, the threshold intensity is larger than $7.4 \mathrm{TW} / \mathrm{cm}^{2}$, and there is no ring

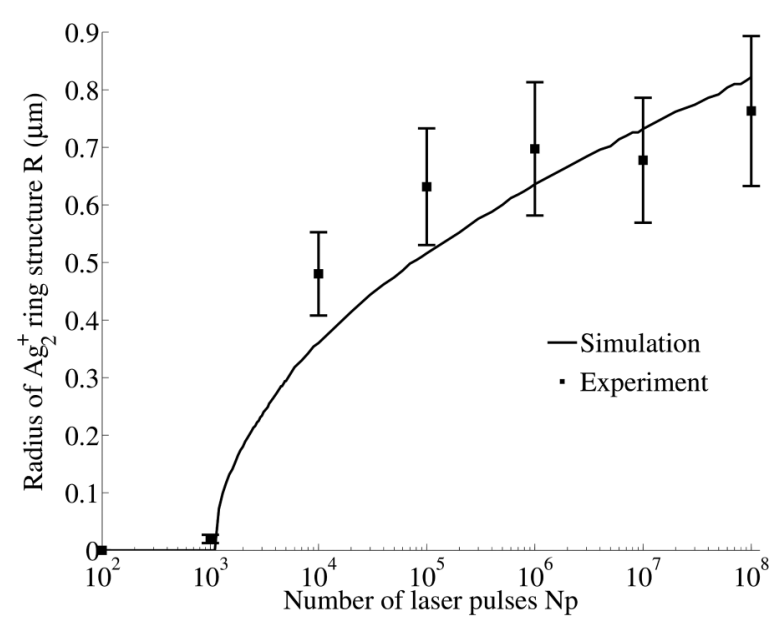

FIG. 8. Radius of ring nanostructure $R$ as a function of the number of laser pulses $N_{p}$ from experiments [5] and numerically predicted. Experiment [5] and numerical simulation. Laser pulse parameters: input pulse intensity is $I_{0}=7.4 \mathrm{TW} / \mathrm{cm}^{2}$, corresponding pulse energy is $E_{0}=167.5 \mathrm{~nJ}$, number of pulses $N_{p}=10^{8}$. Beam waist is $1.2 \mu \mathrm{m}$, pulse duration is $470 \mathrm{fs}$, and the repetition rate is $10 \mathrm{MHz}$. Dissociation function parameters are $S_{0}=80, N=6$. 
structure. But, when the number of pulses is $10^{4}$, the threshold intensity is smaller than $7.4 \mathrm{TW} / \mathrm{cm}^{2}$ because of the cumulative effect, that provides the formation of the on-axis dip in the concentration of the silver clusters $n_{\mathrm{Ag}_{2}}{ }^{+}$. During the first $10^{4}$ pulses, the radius of the nanostructure grows strongly, when after $10^{5}$ laser pulses the growing rate of the radius $R$ decreases significantly because of the fast decay of the dissociation function (Fig. 5, $K_{\text {diss }}$ vanishes for too large radii).

Numerically obtained dependencies of silver ring structure radius with respect to the number of laser pulses, and input pulse intensities, are in a good agreement with the experimental data. Thus, the probability of the dissociation process and the cumulative effect of dissociation during the laser pulse train play a key role in the definition of the silver cluster ring structure geometry.

\section{E. Formation of a local electric field}

Using the full model approach, we are able to reproduce the mechanism of the frozen electric field $E_{\mathrm{dc}}$ formation inside the glass during the DLW. The static electric field reaches a value about $E_{\mathrm{dc}}=10^{9} \mathrm{~V} / \mathrm{m}$ that was estimated using the experimentally measured second harmonic signal [6]. Such large electric field $E_{\mathrm{dc}}$ is already close to the electrical breakdown threshold in phosphate glass, that is about $10^{10}$ $\mathrm{V} / \mathrm{m}[43,47]$. The spatial distribution of charges $Q(r)$ needed to create such a strong electrostatic field comes from the difference in the $\mathrm{Ag}_{2}{ }^{+}$and $\mathrm{Ag}^{2+}$ distributions according to Eq. (18). If the static permittivity $\epsilon_{\mathrm{dc}}$ of the glass is set to unity, the corresponding not-compensated displaced charge density $Q(r)$ is of the order $10^{-4} \times N_{\mathrm{Ag}^{+}}^{0}=10^{17} \mathrm{~cm}^{-3}$ on the length scales inside the silver clusters structure to create $E_{\mathrm{dc}}=10^{9} \mathrm{~V} / \mathrm{m}$. To reach such high values of charge separation, the electron diffusion coefficient $D_{e}$ has to be of the order $1 \times 10^{-3} \mathrm{~m}^{2} \mathrm{~s}^{-1}$.

Thus, the value of the electric field $E_{\mathrm{dc}}$ depends on the rates of kinetic reactions between the silver components, electrons, and holes, on the mobilities of the involved charge species, and on the electrons' drift velocity saturation effect in the strong static electric field. The mechanism of such electron drift velocity saturation in considered process is similar to the charge carrier saturation of the drift velocity in semiconductors under external applied electric field with values higher than $10^{5}-10^{6} \mathrm{~V} / \mathrm{m}$ [46].

The presented results should be considered as a preliminary description of the appearance of a static electric field during the laser-induced metallic clusters formation in the bulk glass. The impact of dipole or bond orientations under a strong orienting electrostatic field $\left(E_{\mathrm{dc}}>10^{7} \mathrm{~V} / \mathrm{m}\right)$ on the creation of the second-order susceptibility $[48,49]$ can lead to an overestimation of the static electric field obtained using the second harmonic signal recording, as it was done in [7]. The possibility of presence of such an orientation mechanism is not clear in described process and it could be investigated experimentally based on the second harmonic polarization analysis [50]. Breaking the centrosymmetry and creating the nonvanished microscopic molecular second-order hyperpolarizability by the applied static electric field, the corresponding second-order nonlinear response of the glass is proportional to $1 / T$, that comes from the orientational averaging using Maxwell-Boltzmann distribution function, and that is needed to go from microscopic hyperpolarizability to macroscopic susceptibility [48]. Thus, we suggest that the addition to the glass susceptibility from the dipoles reorientation does not play an important role in the second harmonic generation (SHG) process in our case because the SHG signal does not change with the temperature $T$ up to the glass transition temperature $T_{g}$ [7]. Additionally, the experimentally observed factor 9-to-1 for SHG intensity for incident polarizations of the probe fields provides a strong evidence of frozen electric field electro-optics assisted SHG since such a ratio would most likely be different for an SHG process resulting from orientation considerations. We can conclude that our SHG process is dominated by electric-field-induced SHG, which does not exclude a minor contribution of orientation rearrangements [50].

According to our simulations, the amplitude of the static electric field $E_{\mathrm{dc}}$ grows rapidly during the first tens of laser pulses. The rate of $E_{\mathrm{dc}}$ increase starts to be smaller after 100 pulses and, after 1000 laser pulses the $E_{\mathrm{dc}}$ amplitude saturates, which is in a good agreement with the experimental observations [6]. This ambipolar electric field $E_{\mathrm{dc}}$ appears because the laser-induced free electrons diffuse faster than holes, and provides the drift of the mobile charged species to compensate diffusion-induced charge separation. Thus, the radial distributions of trapped electrons $\left(\mathrm{Ag}_{2}{ }^{+}\right)$and holes $\left(\mathrm{Ag}^{2+}\right)$ are close to each other and different silver species do not form the coaxial rings structure as it is in other glasses [10,51]. All discussed in our model silver species exist in the same ring structure in case of considered phosphate glass.

Further investigations will focus on a quantitative reproduction of the experimentally observed distribution of the static electric field $E_{\mathrm{dc}}$ during the metallic nanostructures formation.

\section{DISCUSSION AND CONCLUSION}

In general, the material parameters, kinetic rates of clusterization, dissociation pathways and dissociation energies of clusters, diffusion, and mobility of the metallic species define the final distribution of clusters. The general model presented in Sec. II could be used for simulations of femtosecond laser pulse train interaction with other glasses doped by metallic ions. For example, in silicate glass doped by silver ions, the nanoparticle formation occurs under $800-\mathrm{nm}, 1-\mathrm{kHz}$, femtosecond laser irradiation together with further annealing [52]. Glasses containing transition-metal ions from column 11 of the periodic classification are promising candidates for DLW.

$\mathrm{Cu}$ nanoparticles can be formed in silicate and borosilicate glasses under 800-nm, 1-kHz, femtosecond laser irradiation and further annealing [52,53]. This process of $\mathrm{Cu}$ nanoparticle formation is quite close to nanoparticle formation in Ag-doped glass [52]. We can suppose a similar behavior of copper species in the glass under high-repetition rate femtosecond laser irradiation, as compared to silver-containing glasses, even if one has to take into account the $\mathrm{Cu}^{2+}$ absorption spectrum in the near IR which could induce a strong and unwanted absorption of near-IR-laser wavelength [54,55].

In conclusion, we have demonstrated experimentally the similarity of nanostructure formation in various silver-containing glasses. According to these experimental 
observations, we have presented a theoretical model of metallic nanostructure formation in doped glass under a train of high-power femtosecond laser pulses. We numerically investigated the scenario of submicron ring structure formation in silver-doped phosphate glass irradiated by the near-IR femtosecond laser pulses with $10-\mathrm{MHz}$ repetition rate. We reported the glass temperature distribution, that remains below the glass transition temperature of the phosphate matrix in the laser-induced heating process. We presented the mechanism of static electric field creation during the metallic nanostructure formation based on the space charge separation due to the interplay of diffusion and kinetic reactions of involved particles: $e^{-}, h^{+}, \mathrm{Ag}^{+}, \mathrm{Ag}^{0}$. The radial distributions of silver clusters $\mathrm{Ag}_{2}{ }^{+}$obtained numerically are compared with the experimentally observed radial distributions of fluorescence signal of silver clusters. The numerically reproduced radius of silver ring structures is in a good agreement with experimental data in the range of input laser pulse intensities and number of laser pulses in the train that are used for direct laser writing. Our model can be used in general for transparent optical glasses doped by group-11 metals taking into account corresponding metal ion mobilities. The formation of metallic nanostructures during the irradiation process by laser pulses can be predicted for different wavelengths, repetition rates, and input beam geometries, under conditions of nondestructive laser-induced heating of the glass.

\section{ACKNOWLEDGMENTS}

This study has been carried out with financial support from the French State, managed by the French National Research Agency (ANR) in the frame of the Investments for the future Programme IdEx Bordeaux LAPHIA (Grant No. ANR-10-IDEX-03-02). V. Tikhonchuk is acknowledged for his comments regarding this paper.

\section{APPENDIX: DISSOCIATION OF SILVER CLUSTERS}

As it was described by our simulations, the dissociation process of silver clusters plays a key role in ring-shaped nanostructure formation. So, hereafter is discussed a possible way to use a theoretical approach for the photodissociation function based on such parameters as the dissociation energy of the clusters, intensity of the laser pulse, and the energy of photons. The experimentally observed fragmentation pathways of group-11 metals irradiated by UV laser pulses show that the neutral monomer or dimer evaporation is the most possible way of dissociation reaction [56,57]. In our case, this reaction is $\mathrm{Ag}_{2}{ }^{+} \rightarrow \mathrm{Ag}^{+}+\mathrm{Ag}^{0}$. The obtained dissociation energy of this reaction is $E_{D}=1.74 \mathrm{eV}[39,58]$. So, for $\mathrm{UV}$ radiations, the energy of one single photon is enough to provide the dissociation of $\mathrm{Ag}_{2}{ }^{+}$. With near-IR radiation, $\lambda_{0}=1030 \mathrm{~nm}$, the energy of photon is $\hbar \omega=1.2 \mathrm{eV}$, which requires at least two photons for the dissociation process depending on the value of dissociation energy of silver clusters with different number of atoms. The multiphoton dissociation process can be described by the kinetic equation

$$
\frac{\partial n_{\mathrm{Ag}_{2}+}(r, t)}{\partial t}=-\sigma_{K_{d}} \frac{I^{K_{d}}(r, t)}{K_{d} \hbar \omega} n_{\mathrm{Ag}_{2}{ }^{+}},
$$

where $K_{d}=2$ is the multiphoton order of dissociation process, $\sigma_{K_{d}}$ the multiphoton dissociation cross section. We take the value for dissociation cross section equal to $\sigma_{2_{d}}=3 \times$ $10^{-40} \mathrm{~m}^{4} \mathrm{~W}^{-1}$ as a fitting parameter. To calculate the concentration of the silver cluster $\mathrm{Ag}_{2}{ }^{+}$that is dissociated by one laser pulse, we integrate the equation (A1) during the laser pulse propagation:

$$
\Delta n_{\mathrm{Ag}_{2}+}(r)=\int_{-\infty}^{+\infty} n_{\mathrm{Ag}_{2}+} \frac{I^{2}(r, \tau)}{2 \hbar \omega} \sigma_{2_{d}} d \tau .
$$

As an example, for model input distribution of silver clusters $n_{\mathrm{Ag}_{2}+}^{0}(r)=n_{e}^{0} e^{-K r^{2} / a_{0}^{2}}$, the distribution of silver clusters after one iteration of laser-induced dissociation is $n_{\mathrm{Ag}_{2}}+(r)=$ $n_{\mathrm{Ag}_{2}+}^{0}(r)-\Delta n_{\mathrm{Ag}_{2}+}(r)$ and in this case the photodissociation function can be defined as

$$
K_{d i s s}(r)=\Delta n_{\mathrm{Ag}_{2}+}(r) / n_{\mathrm{Ag}_{2}+}^{0}(r) .
$$

The two-photon dissociation function $K_{d i s s}(r)$ of $\mathrm{Ag}_{2}{ }^{+}$is presented in Fig. 9 (top).

For the smallest considered input laser pulse energy $E_{0}=$ $100 \mathrm{~nJ}$, the photodissociation process is not strong enough to form the ring structure of the cluster distribution after one pulse. For input energies $E_{0}=100 \mathrm{~nJ}, 150 \mathrm{~nJ}$, the accumulation along the pulse train is needed to form the on-axis minimum in the cluster distribution. The smaller the energy, the larger number of pulses is needed to produce the ring structure of silver clusters. The dissociation coefficient in the case of smaller energies is not saturated (meaning that it is significantly smaller than 1) [Fig. 9 (top), blue, green, red lines], that is similar to that we took in the numerical simulations (Fig. 5, blue, green, red lines).

For input energy $E_{0}=200 \mathrm{~nJ}$, the on-axis dip in the silver cluster concentration appears already after the first laser pulse, and the maximum value of the cluster concentration is reached at the radial coordinate $r \approx 0.5 \mu \mathrm{m}$ [Fig. 9 (bottom), red line].
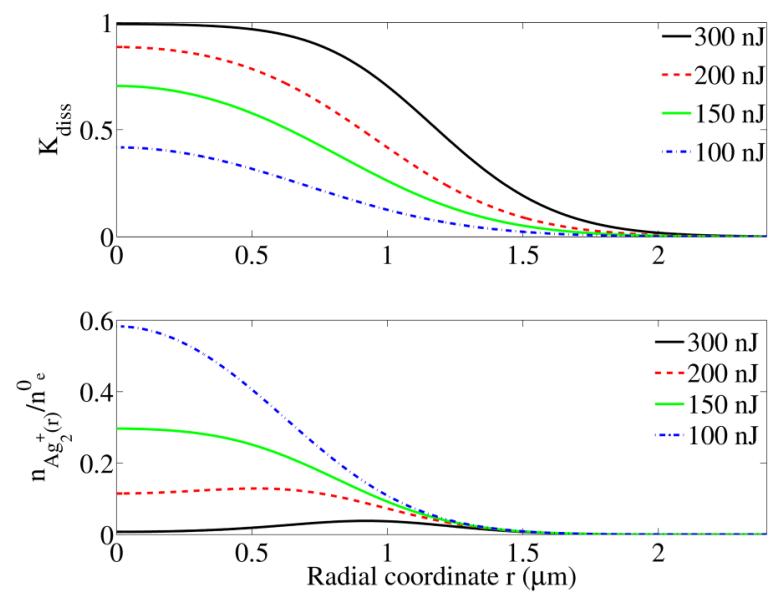

FIG. 9. (Top) The two-photon photodissociation function $K_{\text {diss }}(r)$ of silver cluster $\mathrm{Ag}_{2}{ }^{+}$for number of input laser pulse energies: $E_{0}=100,150,200,300 \mathrm{~nJ}$ calculated according to Eq. (A3). (Bottom) The model radial distribution of the silver clusters $n_{\mathrm{Ag}_{2}+}(r)$ after one iteration of dissociation process. The dissociation energy is $E_{D}=1.74 \mathrm{eV}$, laser pulse wavelength is taken according to the discussed laser pulse parameters: $\lambda_{0}=1030 \mathrm{~nm}$, duration is $470 \mathrm{fs}$, and the beam waist is $1.2 \mu \mathrm{m}$. 
This effect is stronger in case of the highest considered energy $E_{0}=300 \mathrm{~nJ}$ : the ring structure of silver clusters appears with well-resolved on-axis minimum and radial maximum of the ring [Fig. 9 (bottom), black line]. For this energy [Fig. 9 (top), black line] the dissociation coefficient is already saturated; it has the on-axis region with values close to 1 . The "steplike" form in the case of the considered two-photon dissociation process is not so steep as it was taken in the numerical simulations (Fig. 5, black line). Thus, we can suppose that the three-photon dissociation process also occurs. For example, together with discussed $\mathrm{Ag}_{2}{ }^{+}$, the silver clusters with higher number of atoms could exist in the silver nanostructure. The dissociation energy of clusters with odd number of atoms reaches the values about $E_{D}=2.7 \mathrm{eV}$ [39]. So, three photons are needed for the dissociation process. Taking $K_{d}=3$ and the value for dissociation cross section $\sigma_{3_{d}}$ about $4 \times$ $10^{-57} \mathrm{~m}^{6} \mathrm{~W}^{-2}$, the example solution of Eq. (A1) has a similar behavior as the solution in case of two-photon absorption (Fig. 9) taking into account the different multiphoton order of dissociation processes. With a further increase of the silver atoms' number in the cluster, its dissociation energy alternates depending on even or odd number of atoms in a cluster [39].

At the same time, the temperature of the sample influences on the dissociation process of silver clusters [59-61]. So, we have to consider the laser-induced thermal heating process, that provides the increasing of the glass temperature up to values that could significantly influence on the dissociation cross section and on the dissociation energy $E_{D}$ together with the preventing of cluster formation under sufficiently high temperatures. Possibly, we could suppose that the decrease of the dissociation energy is similar to the decrease of the medium band gap under the heating process [62-66], which could be estimated in terms of phonon-assisted ionization process.

Numerically, we compared the distribution of $\mathrm{Ag}_{2}{ }^{+}$that is formed under two different dissociation processes discussed above [pathways (26), (27)]. The process of the ring structure formation and the parameters of the ring structures are quite the same in both cases. The main differences are (1) higher values of the silver cluster concentration $n_{\mathrm{Ag}_{2}+}$ in the on-axis region and (2) smaller values of the static electric field $E_{\mathrm{dc}}$ (which corresponds to a lower amount of separated charges) in the case of dissociation pathway (27). Both effects take place because of the higher population of $\mathrm{Ag}^{0}$ and the absence of additional population of electrons $e^{-}$after the dissociation process according to the pathway (27) in comparison with the pathway (26) used in the full model. In general, both of the pathways could take place depending on the probability of the silver atom formation from the localized pair of silver ion and electron.

Finally, to conclude this discussion, it is important to mention that any of the presented dissociation processes could take place. So, we have chosen to use a phenomenological description of the dissociation function.
[1] R. Gattass, L. Cerami, and E. Mazur, Opt. Express 14, 5279 (2006).

[2] R. Gattass and E. Mazur, Nat. Photonics 2, 219 (2008).

[3] M. Bellec, A. Royon, B. Bousquet, K. Bourhis, M. Treguer, T. Cardinal, M. Richardson, and L. Canioni, Opt. Express 17, 10304 (2009).

[4] A. Royon, Y. Petit, G. Papon, M. Richardson, and L. Canioni, Opt. Mater. Express 1, 866 (2011).

[5] M. Bellec, A. Royon, K. Bourhis, J. Choi, B. Bousquet, M. Treguer, T. Cardinal, J.-J. Videau, M. Richardson, and L. Canioni, J. Phys. Chem. C 114, 15584 (2010).

[6] G. Papon, Y. Petit, N. Marquestaut, A. Royon, M. Dussauze, V. Rodriguez, T. Cardinal, and L. Canioni, Opt. Mater. Express 3, 1855 (2013).

[7] G. Papon, N. Marquestaut, Y. Petit, A. Royon, M. Dussauze, V. Rodriguez, T. Cardinal, and L. Canioni, J. Appl. Phys. 115, 113103 (2014).

[8] J. Choi, M. Bellec, A. Royon, K. Bourhis, G. Papon, T. Cardinal, L. Canioni, and M. Richardson, Opt. Lett. 37, 1029 (2012).

[9] N. Marquestaut, Y. Petit, A. Royon, P. Mounaix, T. Cardinal, and L. Canioni, Adv. Funct. Mater. 24, 5824 (2014).

[10] Y. Dai, G. Yu, M. He, H. Ma, X. Yan, and G. Ma, Appl. Phys. B 103, 663 (2011).

[11] M. Shimizu, M. Sakakura, M. Ohnishi, M. Yamaji, Y. Shimotsuma, K. Hirao, and K. Miura, Opt. Express 20, 934 (2012).

[12] L. Canioni, M. Bellec, A. Royon, B. Bousquet, and T. Cardinal, Opt. Lett. 33, 360 (2008).

[13] M. Falconieri, G. Salvetti, E. Cattaruzza, F. Gonella, G. Mattei, P. Mazzoldi, M. Piovesan, G. Battaglin, and R. Polloni, Appl. Phys. Lett. 73, 288 (1998).
[14] P. Zijlstra, J. W. M. Chon, and M. Gu, Nature (London) 459, 410 (2009).

[15] R. Espiau de Lamaestre, H. Béa, H. Bernas, J. Belloni, and J. L. Marignier, Phys. Rev. B 76, 205431 (2007).

[16] K. Bourhis, A. Royon, M. Bellec, J. Choi, A. Fargues, M. Treguer, J.-J. Videau, D. Talaga, M. Richardson, T. Cardinal, and L. Canioni, J. Non-Cryst. Solids 356, 2658 (2010).

[17] K. Mishchik, Y. Petit, E. Brasselet, A. Royon, T. Cardinal, and L. Canioni, Opt. Lett. 40, 201 (2015).

[18] Y. Petit et al. (unpublished).

[19] A. Delestre, M. Lahaye, E. Fargin, M. Bellec, A. Royon, L. Canioni, M. Dussauze, F. Adamietz, and V. Rodriguez, Appl. Phys. Lett. 96, 091908 (2010).

[20] E. Janata, A. Henglein, and B. Ershov, J. Phys. Chem. 98, 10888 (1994).

[21] E. Janata, Res. Chem. Intermed. 27, 733 (2001).

[22] N. Bulgakova, R. Stoian, A. Rosenfeld, I. Hertel, W. Marine, and E. Campbell, Appl. Phys. A 81, 345 (2005).

[23] N. M. Bulgakova, R. Stoian, A. Rosenfeld, I. V. Hertel, and E. E. B. Campbell, Phys. Rev. B 69, 054102 (2004).

[24] S. Anvari, C. Hogarth, and G. Moridi, J. Mater. Sci. 26, 3639 (1991).

[25] L. V. Keldysh, J. Exptl. Theoret. Phys. (USSR) 47, 1945 (1964) [Sov. Phys.-JETP 20, 1307 (1965)].

[26] B. C. Stuart, M. D. Feit, S. Herman, A. M. Rubenchik, B. W. Shore, and M. D. Perry, Phys. Rev. B 53, 1749 (1996).

[27] D. Arnold and E. Cartier, Phys. Rev. B 46, 15102 (1992).

[28] D. F. Price, R. M. More, R. S. Walling, G. Guethlein, R. L. Shepherd, R. E. Stewart, and W. E. White, Phys. Rev. Lett. 75, 252 (1995). 
[29] A. Couairon, L. Sudrie, M. Franco, B. Prade, and A. Mysyrowicz, Phys. Rev. B 71, 125435 (2005).

[30] J. Rolle, L. Bergé, G. Duchateau, and S. Skupin, Phys. Rev. A 90, 023834 (2014).

[31] K. Bourhis, Photostructuration par laser infrarouge femtoseconde de verres photosensibles de phosphates de zinc, d'argent et de gallium, Ph.D. thesis, University of Bordeaux 1, 2011.

[32] A. Royon, K. Bourhis, L. Bchou, T. Cardinal, L. Canioni, and Y. Deshayes, Microelectron. Reliab. 53, 1514 (2013).

[33] M. Bellec, Nanostructuration par laser femtoseconde dans un verre photo-luminescent, Ph.D. thesis, University of Bordeaux 1, 2009.

[34] L. B. Fletcher, J. J. Witcher, N. Troy, S. T. Reis, R. K. Brow, and D. M. Krol, Opt. Express 19, 7929 (2011).

[35] O. L. Anderson and D. A. Stuart, J. Am. Ceram. Soc. 37, 1551 (1954).

[36] G. V. Buxton, Compilation of Rate Constant for the Reaction of Metal Ions in Unusual Valence States (National Bureau of Standards (U. S.), Washington, 1978).

[37] P. Atkins and J. de Paula, Physical Chemistry for the Life Sciences (Oxford University Press, Oxford, UK, 2011).

[38] Y. Petit, K. Mishchik, N. Varkentina, N. Marquestaut, A. Royon, I. Manek-Honninger, T. Cardinal, and L. Canioni, Opt. Lett. 40, 4134 (2015).

[39] S. Kruckeberg, G. Dietrich, K. Lutzenkirchen, L. Schweikhard, C. Walther, and J. Ziegler, J. Chem. Phys. 110, 7216 (1999).

[40] G. Gruener, P. Odier, D. De Sousa Meneses, P. Florian, and P. Richet, Phys. Rev. B 64, 024206 (2001).

[41] D. Griffiths, Introduction to Electrodynamics (Prentice Hall, Englewood Cliffs, NJ, 1999).

[42] S. Honkanen, B. West, S. Yliniemi, P. Madasamy, M. Morrell, J. Auxier, A. Schlzgen, N. Peyghambarian, J. Carriere, J. Frantz, R. Kostuk, J. Castro, and D. Geraghty, Physics and Chemistry of Glasses: European Journal of Glass Science and Technology Part B 47, 110 (2006).

[43] C. R. Phipps, Laser Ablation and its Applications, 1st ed. (Springer, New York, 2007).

[44] T. Itina, O. Uteza, N. Sanner, and M. Sentis, J. Optoelectron. Adv. Mater. (JOAM) 12, 470 (2010).

[45] L. Jiang and H. Tsai, Int. J. Heat Mass Transfer 48, 487 (2005).

[46] P. Yu and M. Cardona, Fundamentals of Semiconductors: Physics and Materials Properties (Springer, Berlin, 2010).
[47] F. Wooten, Phys. Lett. A 55, 255 (1975).

[48] N. Mukherjee, R. A. Myers, and S. R. J. Brueck, J. Opt. Soc. Am. B 11, 665 (1994).

[49] R. A. Myers, N. Mukherjee, and S. R. J. Brueck, Opt. Lett. 16, 1732 (1991).

[50] P. Kazansky and P. Russel, Opt. Commun. 110, 611 (1994).

[51] Y. Liu, M. Shimizu, B. Zhu, Y. Dai, B. Qian, J. Qiu, Y. Shimotsuma, K. Miura, and K. Hirao, Opt. Lett. 34, 136 (2009).

[52] B. Hua, Y. Shimotsuma, M. Nishi, K. Miura, and K. Hirao, J. Laser Micro Nanoen. 2, 36 (2007).

[53] J. M. P. Almeida, L. D. Boni, W. Avansi, C. Ribeiro, E. Longo, A. C. Hernandes, and C. R. Mendonca, Opt. Express 20, 15106 (2012).

[54] B.-S. Bae and M. C. Weinberg, J. Non-Cryst. Solids 168, 223 (1994).

[55] S. Bruni, F. Cariati, and D. Narducci, Vib. Spectrosc. 7, 169 (1994).

[56] C. Walther, S. Becker, G. Dietrich, H.-J. Kluge, M. Lindinger, K. Ltzenkirchen, L. Schweikhard, and J. Ziegler, Z. Phys. D: At., Mol. Clusters 38, 51 (1996).

[57] M. Vogel, A. Herlert, and L. Schweikhard, J. Am. Soc. Mass Spectrom. 14, 614 (2003).

[58] M. Vogel, K. Hansen, A. Herlert, and L. Schweikhard, Phys. Rev. Lett. 87, 013401 (2001).

[59] Q.-Z. Zhao, J.-R. Qiu, X.-W. Jiang, C.-J. Zhao, and C.-S. Zhu, Opt. Express 12, 4035 (2004).

[60] K. Bourhis, A. Royon, G. Papon, M. Bellec, Y. Petit, L. Canioni, M. Dussauze, V. Rodriguez, L. Binet, D. Caurant, M. Treguer, J.-J. Videau, and T. Cardinal, Mater. Res. Bull. 48, 1637 (2013).

[61] T. Yoshino, Y. Ozeki, M. Matsumoto, and K. Itoh, Jpn. J. Appl. Phys. 51, 102403 (2012).

[62] W. S. Chow, Phys. Rev. 185, 1056 (1969).

[63] K. Saito and A. J. Ikushima, Phys. Rev. B 62, 8584 (2000).

[64] A. Belsky, P. Martin, H. Bachau, A. N. Vasilev, B. N. Yatsenko, S. Guizard, G. Geoffroy, and G. Petite, Europhys. Lett. 67, 301 (2004).

[65] H. X. Deng, X. Xiang, W. G. Zheng, X. D. Yuan, S. Y. Wu, X. D. Jiang, F. Gao, X. T. Zu, and K. Sun, J. Appl. Phys. 108, 103116 (2010).

[66] G. Duchateau, M. D. Feit, and S. G. Demos, J. Appl. Phys. 115, 103506 (2014). 